\title{
Structure-function relationships of HDL in diabetes and coronary heart disease
}

\author{
Mathias Cardner, ${ }^{1,2}$ Mustafa Yalcinkaya, ${ }^{3}$ Sandra Goetze, ${ }^{4,5}$ Edlira Luca, ${ }^{6}$ Miroslav Balaz, ${ }^{4}$ \\ Monika Hunjadi, ${ }^{7}$ Johannes Hartung, ${ }^{8}$ Andrej Shemet, ${ }^{9}$ Nicolle Kränkel, ${ }^{8}$ Silvija Radosavljevic, ${ }^{3}$ \\ Michaela Keel, ${ }^{3}$ Alaa Othman, ${ }^{3}$ Gergely Karsai, ${ }^{3}$ Thorsten Hornemann, ${ }^{3}$ Manfred Claassen, ${ }^{5,10}$ \\ Gerhard Liebisch, ${ }^{11}$ Erick Carreira, ${ }^{9}$ Andreas Ritsch, ${ }^{7}$ Ulf Landmesser, ${ }^{8}$ Jan Krützfeldt, ${ }^{6}$ \\ Christian Wolfrum, ${ }^{4}$ Bernd Wollscheid, ${ }^{4,5}$ Niko Beerenwinkel, ${ }^{1,2}$ Lucia Rohrer, ${ }^{3}$ \\ and Arnold von Eckardstein ${ }^{3}$
}

'Department of Biosystems Science and Engineering, Swiss Federal Institute of Technology in Zurich (ETH Zurich), Basel, Switzerland. ${ }^{2}$ Swiss Institute of Bioinformatics (SIB), Basel, Switzerland. ${ }^{3}$ Institute of Clinical Chemistry, University of Zurich and University Hospital of Zurich, Zurich, Switzerland. ${ }^{4}$ Department of Health Sciences and Technology and 5 Institute of Molecular Systems Biology, ETH Zurich, Zurich, Switzerland. ${ }^{6}$ Department of Diabetology and Endocrinology, University of Zurich and University Hospital of Zurich, Zurich, Switzerland. 'Department of Internal Medicine, Medical University of Innsbruck, Innsbruck, Austria. ${ }^{8}$ Department of Cardiology, University Medicine Charité Berlin, Berlin, Germany. ${ }^{9}$ Department of Chemistry and Applied Biosciences and ${ }^{10}$ Department of Biology, ETH Zurich, Zurich, Switzerland. "Institute of Clinical Chemistry and Laboratory Medicine, University Hospital Regensburg, Regensburg, Germany.

\begin{abstract}
High-density lipoproteins (HDL) contain hundreds of lipid species and proteins and exert many potentially vasoprotective and antidiabetogenic activities on cells. To resolve structure-functiondisease relationships of $\mathrm{HDL}$, we characterized $\mathrm{HDL}$ of 51 healthy subjects and 98 patients with diabetes (T2DM), coronary heart disease (CHD), or both for protein and lipid composition, as well as functionality in $\mathbf{5}$ cell types. The integration of $\mathbf{4 0}$ clinical characteristics, $\mathbf{3 4}$ nuclear magnetic resonance (NMR) features, 182 proteins, 227 lipid species, and 12 functional read-outs by highdimensional statistical modeling revealed, first, that CHD and T2DM are associated with different changes of HDL in size distribution, protein and lipid composition, and function. Second, different cellular functions of HDL are weakly correlated with each other and determined by different structural components. Cholesterol efflux capacity (CEC) was no proxy of other functions. Third, 3 potentially novel determinants of HDL function were identified and validated by the use of artificially reconstituted $\mathrm{HDL}$, namely the sphingadienine-based sphingomyelin SM 42:3 and glycosylphosphatidylinositol-phospholipase $\mathrm{D} 1$ for the ability of HDL to inhibit starvation-induced apoptosis of human aortic endothelial cells and apolipoprotein F for the ability of HDL to promote maximal respiration of brown adipocytes.
\end{abstract}

Authorship note: MC, MY, and SG contributed equally to this work.

Conflict of interest: UL declares the receipt of modest speaker or advisory honorary from Amgen, Sanofi, Medicines Company, and Novartis, as well as research grants from Amgen and Bayer.

Copyright: (c) 2020, American Society for Clinical Investigation.

Submitted: July 1, 2019 Accepted: December 4, 2019 Published: December 12, 2019

Reference information: JCI Insight. 2020;5(1):e131491.

https://doi.org/10.1172/jci.

insight.131491

\section{Introduction}

Low plasma levels of high-density lipoprotein cholesterol (HDL-C) are associated with increased risks of coronary heart disease (CHD) and diabetes mellitus type 2 (T2DM) (1, 2). HDL particles exert diverse potentially antiatherogenic and antidiabetic effects $(3,4)$. Furthermore, atherosclerosis or hyperglycemia could be decreased or even reverted in several animal models by transgenic overexpression or exogenous application of apolipoprotein A-I (apoA-I), the most abundant protein of HDL (3, 4). However, in humans, drugs increasing HDL-C such as fibrates, niacin, and inhibitors of cholesteryl ester transfer protein (CETP) have failed to prevent fatal or nonfatal cardiovascular endpoints $(2,5)$. Moreover, in several inborn errors of human HDL metabolism and genetic mouse models with altered HDL metabolism, low or high HDL-C levels were not associated with any differences in cardiovascular risk and atherosclerotic plaque load, respectively $(2,3)$. Because of these ambiguous data, the causal role and, hence, suitability as a therapeutic target of HDL-C is questioned (2). However, by contrast to the disease-causing cholesterol in LDL (6), the cholesterol in HDL (i.e., HDL-C) neither exerts nor reflects any of the potentially antiatherogenic activities of HDL (3). HDL-C is only a nonfunctional surrogate marker for estimating the HDL pool size without deciphering the heterogeneous composition and, hence, functionality of $\operatorname{HDL}(2,3)$. 
In a prototypic HDL particle, 2-5 molecules of apoA-I and approximately 100 phosphatidylcholine molecules form an amphipathic shell, in which several molecules of unesterified cholesterol are embedded and which surrounds a core of water-insoluble cholesteryl esters and triglycerides. Differences in the molar content of apoA-I and of major lipid constituents cause differences of HDL subclasses in shape, size, and charge (3). HDL particles carry hundreds of different quantitatively minor proteins and lipid species (3), many of which are not just passive cargo (like cholesterol) but are biologically active. This physiological heterogeneity is further increased in HDL of patients - for example, with T2DM or CHD — by the loss or structural modification of typical HDL constituents or by the acquisition of atypical constituents (7). This structural complexity of HDL is accompanied by functional diversity. The classical function of HDL is the stimulation of cholesterol efflux $(2,3,7,8)$. In addition, HDL particles also elicit various cellular signal transduction processes through interactions of specific components with specific receptors - for example, apoM bound sphingosine-1-phosphate (S1P) with S1P receptors $(3,7)$. The resulting changes in cellular cholesterol homeostasis and signaling events, respectively, have been associated with survival, proliferation, and functionality of cells, including macrophages, endothelial cells, pancreatic $\beta$ cells, adipocytes, or myocytes (7). Several of these functions are disturbed in patients with $\operatorname{CHD}$ or T2DM $(7,8)$.

The relative importance of the many physiological and pathological activities of normal and dysfunctional HDL, respectively, for the pathogenesis of atherosclerosis or T2DM is unknown. In a systems biology approach (Figure 1), we characterized the size distribution and the lipid and protein composition of HDL by nuclear magnetic resonance (NMR) spectroscopy and mass spectrometry (MS), as well as the functionality of HDL in 5 cellular systems. Using high-dimensional statistical modeling, specifically probabilistic graphical models, we unraveled several distinct associations of HDL components with T2DM or $\mathrm{CHD}$, as well as cellular functionality of HDL. Finally, as proof of principle, we validated the predicted functional relevance of 2 proteins and 1 lipid species by targeted experiments using reconstituted HDL (rHDL), which were reconstituted with or without the component of interest (Figure 1).

\section{Results}

Associations of structural and functional HDL features with disease status. We characterized the structural composition and functionality of HDL in 51 healthy volunteers, 46 patients with T2DM only, 25 patients with CHD only, and 27 patients with both T2DM and CHD. As expected, patients and healthy volunteers differ significantly by many anthropometric and clinical measures, as well as drug treatment (Supplemental Table 1; supplemental material available online with this article; https://doi.org/10.1172/jci. insight.131491DS1). Supplemental Figures 1-3 show volcano plots of the disease associations of 34 HDL features measured by NMR spectroscopy of plasma, as well as 182 proteins and 227 lipids measured by MS of isolated HDL. Data are adjusted for sex and sampling site, as well as by date and centrifuge used for HDL isolation. All $P$ values are adjusted for multiple testing.

For T2DM, NMR spectroscopy reveals a shift of HDL particle composition with a loss of large and very large particles and the gain of small triglyceride-rich particles. The comparison of CHD patients with healthy control subjects does not reveal any significant difference in HDL particle size or composition (Supplemental Figure 1).

HDLs of patients with CHD or T2DM (HDL ${ }_{\mathrm{CHD}}$ and $\mathrm{HDL}_{\mathrm{T} 2 \mathrm{DM}}$, respectively) differ from HDLs of healthy controls $\left(\mathrm{HDL}_{\text {healthy }}\right)$ by the loss of lipid species: $\mathrm{In}_{\mathrm{HDL}} \mathrm{CHD}$ and $\mathrm{HDL}_{\mathrm{T} 2 \mathrm{DM}}$, concentrations of 5 and 71 lipid species, respectively, are present at significantly lower concentrations. Conversely, only 4 and 14 lipid species occur at significantly higher concentrations in $\mathrm{HDL}_{\mathrm{CHD}}$ and $\mathrm{HDL}_{\mathrm{T} 2 \mathrm{DM}}$, respectively, than in $\mathrm{HDL}_{\text {healthy }}$ (Supplemental Figure 2). Both $\mathrm{HDL}_{\mathrm{CHD}}$ and $\mathrm{HDL}_{\text {т2Dм }}$ are enriched in phosphatidylethanolamines $\mathrm{PE} \mathrm{38:5,} \mathrm{PE} 38: 6$, and $\mathrm{PE}$ 40:7. Also of note, all 5 lipid species decreased in $\mathrm{HDL}_{\mathrm{CHD}}$ are also reduced in $\mathrm{HDL}_{\mathrm{T} 2 \mathrm{DM}}$, namely the phosphatidylinositols PI 36:2 and PI 34:2, the phosphatidylcholines PC 36:2 and PC 34:2, as well as the cholesteryl ester $\mathrm{CE}$ 18:2. In addition, $\mathrm{HDL}_{\mathrm{T} 2 \mathrm{DM}}$ is characterized by strong reductions in the content of ether-phosphatidylcholines (PC O-34:2, PC O-34:3, PC O-36:2, PC O-36:3), lysophosphatidylcholines (LPC 18:2, LPC 18:1, LPC 18:0), and sphingomyelins (most prominently for SM 42:3 and SM 36:3) (Supplemental Figure 2).

$\mathrm{HDL}_{\mathrm{CHD}}$ is characterized by the relative enrichment of specific proteins, whereas $\mathrm{HDL}_{\mathrm{T} 2 \mathrm{DM}}$ is rather deprived of specific proteins. Compared with $\mathrm{HDL}_{\text {healthy }}, 6$ and 4 proteins are present at significantly higher and lower concentrations, respectively, in $\mathrm{HDL}_{\mathrm{CHD}}$ (Supplemental Figure 3). Conversely, in $\mathrm{HDL}_{\mathrm{T} 2 \mathrm{DM}}, 17$ and 44 proteins are present at significantly higher and lower concentrations, respectively (Supplemental Figure 3). Both $\mathrm{HDL}_{\mathrm{CHD}}$ and $\mathrm{HDL}_{\mathrm{T} 2 \mathrm{DM}}$ are deprived of apoA-IV but enriched with pulmonary surfactant 


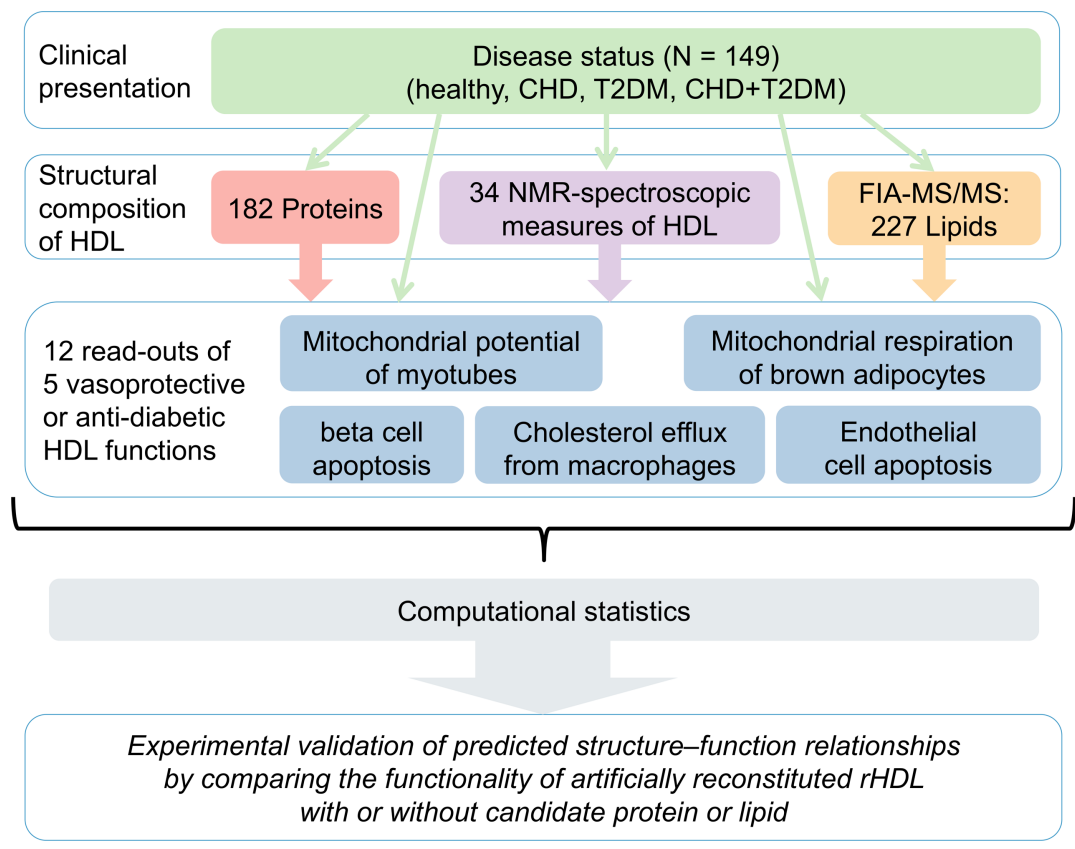

Figure 1. Scheme summarizing the strategy and workflow toward a probabilistic graphical model integrating disease status with structure of $\mathrm{HDL}$ and cellular responses to HDL. FIA-MS/MS, flow injection analysis-tandem mass spectrometry.

protein $\mathrm{B}$ (PSPB), as well as serum amyloid A proteins, SAA1 and SAA2. In addition, $\mathrm{HDL}_{\mathrm{T} 2 \mathrm{DM}}$ is characterized by the loss of clusterin, paraoxonases PON1 and PON3, and apoD, apoE, apoF, and apoM and the gain of fibrinogen and apoC-II and apoC-III.

Supplemental Table 2 provides an overview of the in vitro cell culture models that we used for the functional testing of HDL. In mixed-effects models adjusting for sex, sampling site, date, and centrifuge used for HDL isolation, as well as plate effects, several functions show statistically significant disease associations (Supplemental Figure 4). Effect sizes are presented relative to SDs of the functional read-outs (see Supplemental Methods 5). The ability to promote maximal respiration of brown adipocytes (respiration $\max _{\max }$ ) is increased for $\mathrm{HDL}_{\mathrm{CHD}}(0.68[95 \% \mathrm{CI}, 0.11-1.3])$ but decreased for $\mathrm{HDL}_{\mathrm{T2DM}}(-0.4$ [95\% CI, -0.77 to $-0.02]) . \mathrm{HDL}_{\text {T2DM }}$ is also characterized by reduced ability to inhibit starvation-induced apoptosis of human aortic endothelial cells (HAECs) (apoptosis HAEC $)(0.5$ [95\% CI, 0.15-0.85]). Of note, the CEC of neither isolated HDL nor apoB-free plasma differs significantly between patients and healthy control subjects.

In order to find independent predictors of disease status, we used multiple logistic regression with elastic net regularization, coupled with stability selection. Figure 2 shows associations of T2DM, CHD, or the combination of both with several variables in terms of the regression coefficients (orange and green for positive and inverse associations, respectively) and stability selection (diamonds). The more conservative stability selection reveals expected associations of T2DM, CHD, or both, with clinical measures either defining the disease (e.g., glucose and glycated hemoglobin A1c [HbAlc]) or being risk factors (age, hypertension), or with effects of very well-established treatment (lower LDL-C). Only T2DM or the combination of T2DM with CHD show stable associations with HDL-related features (indicated by diamonds in Figure 2); $\mathrm{HDL}_{\text {T2DM }}$ have a reduced ability to lower mitochondrial potential of myotubes after 2 hours (2hr-mitochondrial membrane potential [2hr-MMP] C2C12), a reduced lipid content and number of large particles, and a reduced content of the IgG FC $\gamma$ fragment binding protein (FCGBP). Also, LPC 22:5 is strongly and inversely associated with T2DM. The combination of T2DM and CHD shows stable positive associations with the content of PSPB, as well as stable negative associations with PI 36:2 and podocalyxin (PODXL). Although weaker and less robust, $\mathrm{HDL}_{\mathrm{T} 2 \mathrm{DM}+\mathrm{CHD}}$ is also characterized by a decreased content in apoE and PON1. LPC 18:0 is the only HDL component that is independently associated with both T2DM and the combination of T2DM and CHD. CHD shows several associations, but no HDL-related feature persists upon stability selection (no diamonds in Figure 2). Among them, the inverse associations with apoA-I and lactate dehydrogenase ( $\mathrm{LDH})$ are the strongest, followed by extracellular matrix protein 1 (ECM1, inverse), apoA-IV (inverse), secretoglobin family $3 \mathrm{~A}$ member 2 


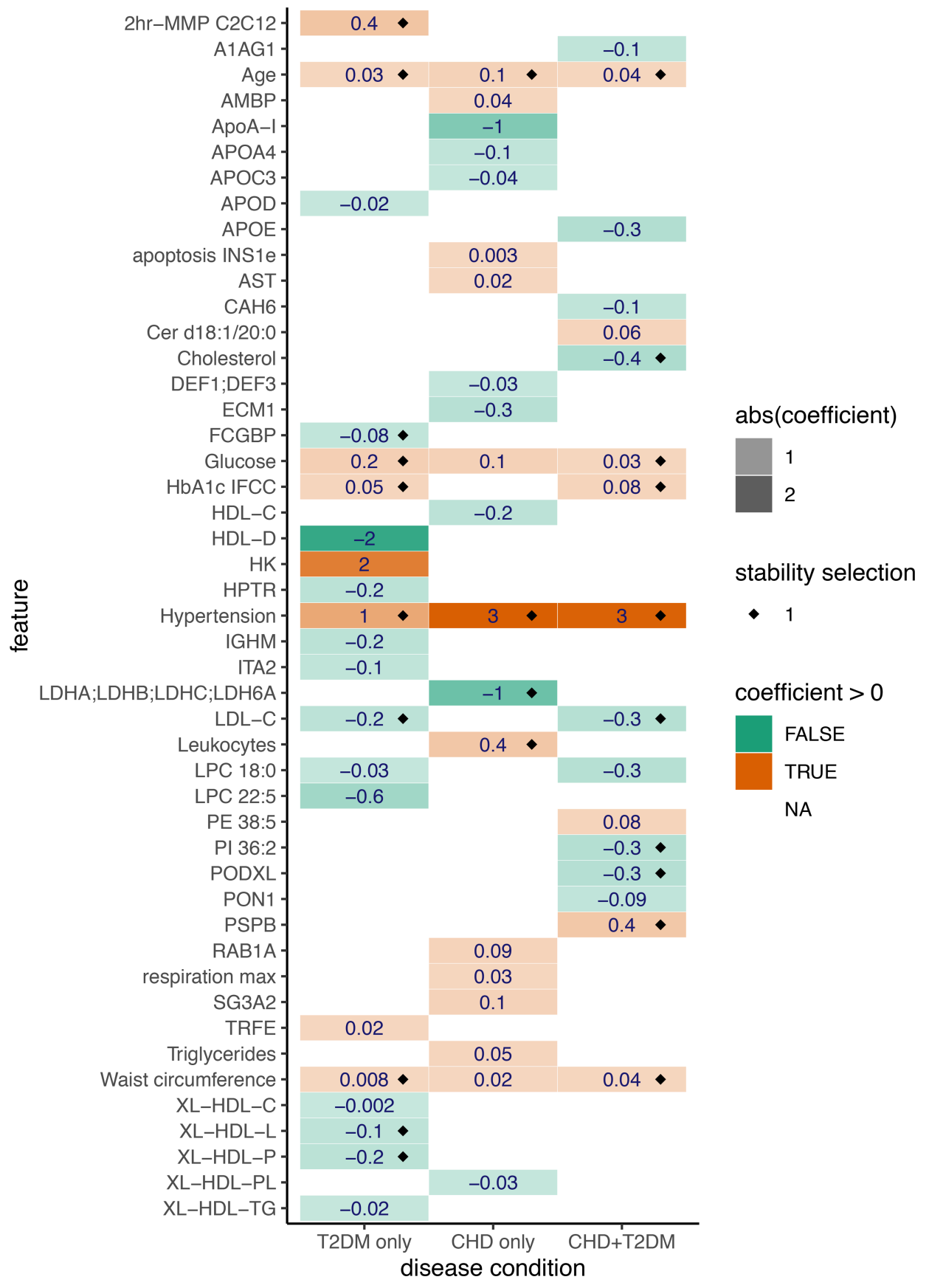

Figure 2. Logistic regressions of disease conditions as explained by clinical covariates, as well as functional and structural features of $\mathrm{HDL}$. Colors indicate the signs of the printed regression coefficients estimated using elastic net regularization. The color intensity reflects the absolute value (magnitude) of a given regression coefficient. White cells correspond to coefficients estimated as zero due to regularization. Diamonds indicate features chosen by stability selection, with fewer than 2 expected false selections per disease condition.

abs(coefficient)

stability selection

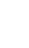




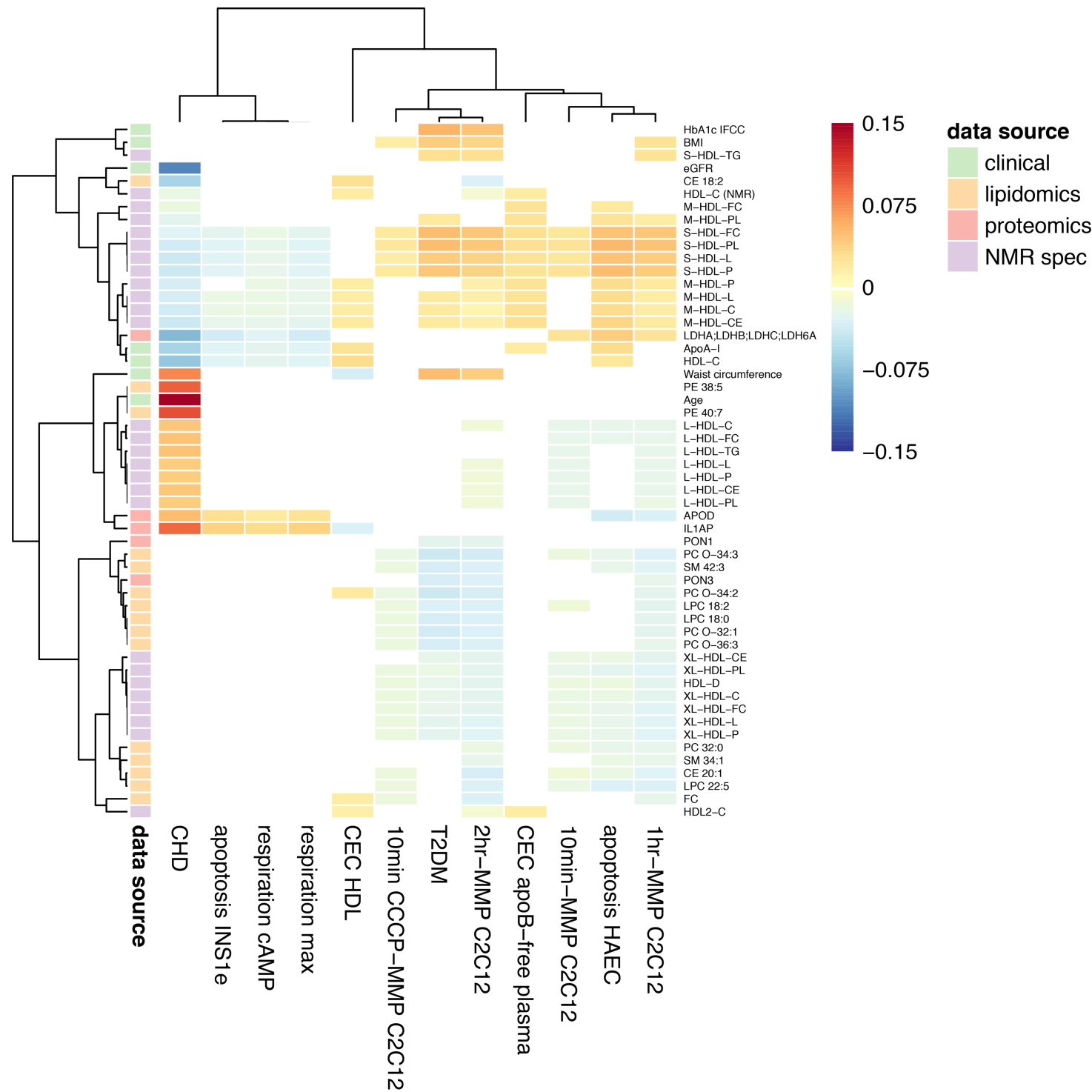

Figure 3. Multivariate analysis of disease status and HDL function regressed on HDL structure and subclasses using sparse partial least squares, adjusted for clinical covariates. The heat map shows the regression coefficients for features (rows) with respect to responses (columns), and the color gradient indicates the magnitude and sign of each coefficient. All features were standardized to have mean zero and variance 1 , and the regression coefficients should be interpreted in a relative rather than an absolute sense. White cells correspond to coefficients, which - due to sparsity constraints - are estimated as zero. Rows and columns are clustered based on correlation distance, thereby indicating similarity of regression coefficient profiles. The color code left of the heatmap indicates the data source of each feature.

apoptosis $_{\mathrm{HAEC}}$, and $\mathrm{CEC}_{\mathrm{apoB}-\text { free plasma }}$. $\mathrm{CHD}$ is most similar to the ability of $\mathrm{HDL}$ to promote respiration of brown adipocytes and apoptosis ${ }_{\mathrm{INS1e}}$. The complex of T2DM/apoptosis $\mathrm{HAEC}_{\mathrm{H}} / \mathrm{MMP}_{\mathrm{C} 2 \mathrm{C} 12}$ is associated with 2 clusters of structural or clinical features; one positively associated cluster encompasses clinical measures of glycemia and body mass, as well as several NMR measures of small or medium-sized HDL. The other inversely associated cluster encompasses measures of very large HDL, as well as the atypical sphingomyelin SM 42:3 and the ether

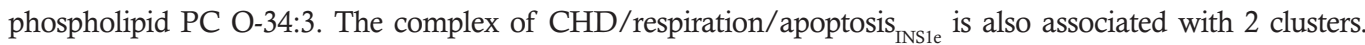
One is positively associated with CHD and characterized by higher activity of HDL to promote mitochondrial respiration. This cluster has virtually no association with T2DM but is associated with age, several NMR measures of large HDL, polyunsaturated phosphatidylethanolamines PE38:5 and PE40:7, apoD, and IL-1 receptor accessory protein IL1AP. The other cluster with inverse CHD association also shows inverse association with respiration. It contains clinical measures of $\mathrm{HDL}$, such as apoA-I and HDL-C, as well as NMR-based measures of HDL-C and small and medium-sized HDL (Figure 3). A part of this cluster is shared between CHD and T2DM, but in opposite directions (Figure 3). 
data source
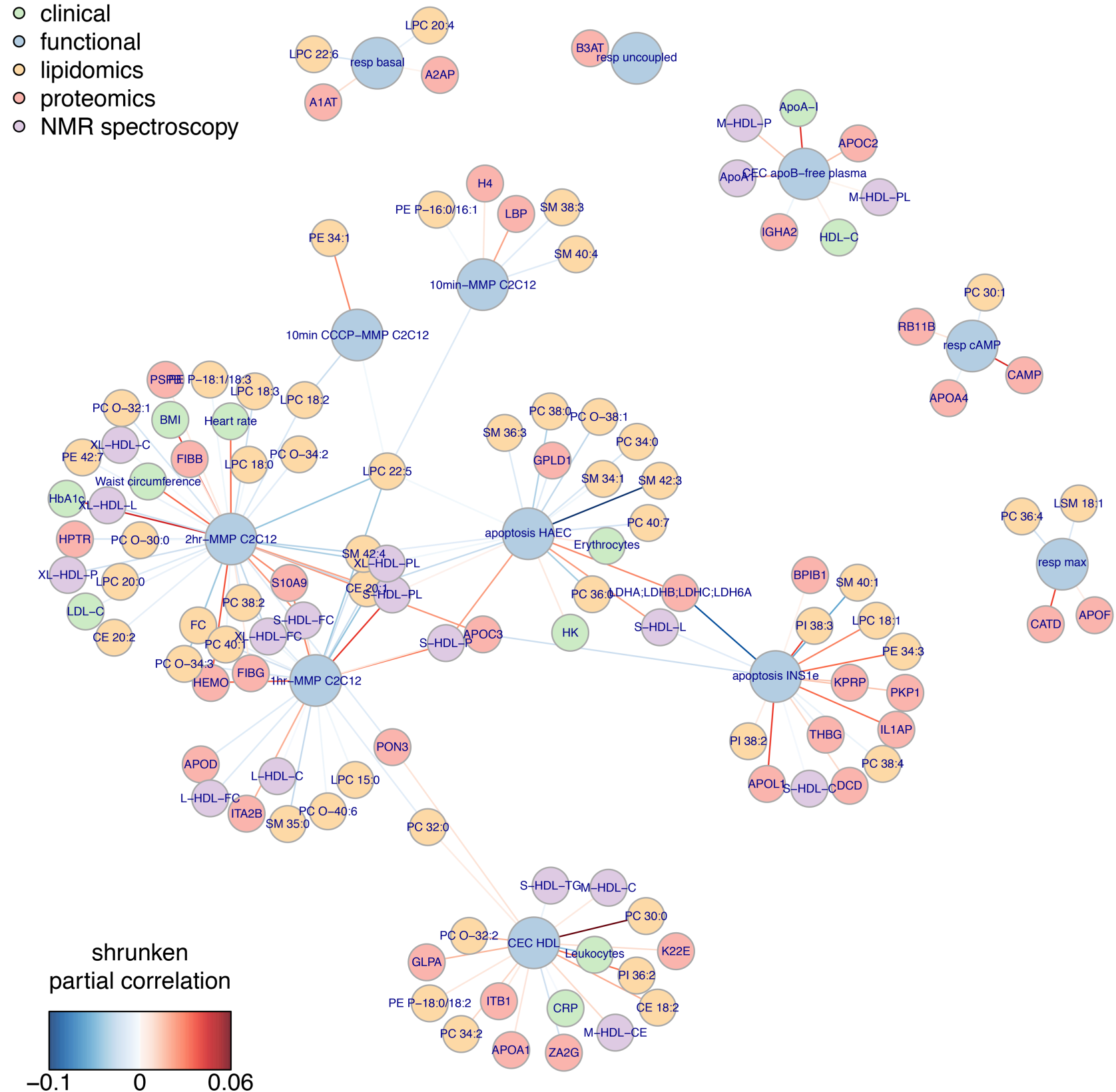

Figure 4. Gaussian graphical model estimating conditional dependencies between HDL function, structure, and subclasses, adjusted for clinical covariates. For legibility, we show only the bipartite subgraph connecting HDL functions to other features. Edges (lines) indicate partial correlations between HDL functions (blue) and proteins (red), lipid species (orange), and abundance of HDL subclasses (violet). Clinical features (green) are included to adjust for potential confounding. The gradient edge color indicates whether the corresponding partial correlation is positive (red) or negative (blue).

Associations of proteins and lipids with functions of HDL. We used a Gaussian graphical model to jointly analyze conditional dependencies between HDL function and structure, while adjusting for potentially confounding clinical covariates. The main result is presented in Figure 4 as a graph in which nodes are clinical, functional, and structural features. Two features are connected by an undirected edge if they are conditionally dependent, given all other features in the model. Most functional read-outs have unique relationships with clinical features, as well as NMR measures, proteins, and lipids of HDL particles. There are only very few examples where 1 parameter is associated with more than 1 function. Those functions are very closely related. For example, apoC-III and S100 protein S10A9 (both positive), LPC 22:5 (negative), as well as free cholesterol (FC) in small HDL (positive) and very large HDL (inverse) connect different time points of $\mathrm{MMP}_{\mathrm{C} 2 \mathrm{C} 12}$ (see also partial correlations depicted in Supplemental Figure 6). 
$\mathrm{CEC}_{\mathrm{HDL}}$ and $\mathrm{CEC}_{\text {apoB-free plasma }}$ are both predominantly associated with differences in apoA-I measured either by the clinical assay, NMR spectroscopy $\left(\mathrm{CEC}_{\text {apoB-free plasma }}\right)$, or proteotype analysis $\left(\mathrm{CEC}_{\mathrm{HDL}}\right)$. Of note, $\mathrm{CEC}_{\text {apoB-free plasma }}$ shows the strongest correlation with the concentration of apoA-I and number of medium-sized HDL particles. $\mathrm{CEC}_{\mathrm{HDL}}$ has more significant correlations - among which, those with PC 30:0, PI 36:2, and CE 18:2 are the strongest (Supplemental Figure 6).

Apoptosis $_{\mathrm{HAEC}}$ shows several correlations with specific lipids of HDL. Among them, the inverse with SM 42:3 is the strongest. LDH (positive) and glycosylphosphatidyl-inositol specific phospholipase D1 (GPLD1,

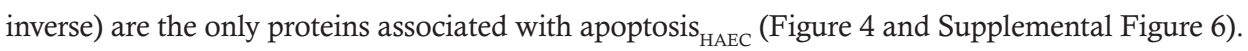

$\mathrm{LDH}$ is also associated with apoptosis INSle $_{\text {but in the opposite direction as apoptosis }}$ HAEC $($ Figure 4 and Supplemental Figure 6). Other significant correlations are seen with SM 40:1 (inverse), PI 38:3, PE 34:3, LPC 18:1, IL1AP, and apoL1 (all positive).

Several NMR measures of small HDL, S100A9, and apoC-III evolve as the strongest positive determinants of $\mathrm{MMP}_{\mathrm{C} 2 \mathrm{C} 12}$. Several glycerophospholipid species are inversely correlated with $\mathrm{MMP}_{\mathrm{C} 2 \mathrm{C} 12}$. Among them, LPC 22:5 is the only measure that shows significant inverse correlations at every time point. Also of note, the phospholipid content of very large and small particles show opposite correlations with $\mathrm{MMP}_{\mathrm{C} 2 \mathrm{C} 12}$ at different time points.

The activity of HDL to promote mitochondrial respiration of brown adipocytes has several determinants, which surprisingly are not shared between the different phases. The strongest determinants include Cathelicidin antimicrobial peptide (CAMP) for respiration cAMP $_{\text {and cathepsin D (CATD) for respiration }}$ max . ApoF is another positive determinant of respiration ${ }_{\max }$. Interestingly, several lipids emerge as negative determinants of mitochondrial respiration. Among them, the strongest correlations are found between LPC 22:6 and respiration basal , as well as between lysosphingomyelin LSM 18:1 or PC36:4 and respiration max $_{\text {. }}$

Experimental validation of structure-function relationships of $H D L$. Finally, we validated some of the structure-function relationships predicted by the graphical model of Figure 4. To this end, we used the cholate dialysis method to generate rHDL consisting of apoA-I and dioleyl-phosphatidylcholine (DOPC) in a molar ratio of 1:100 with or without the protein or lipid of interest. The respective candidate proteins or lipids are included at 3 different amounts, reflecting the mean, lowest, and highest concentration encountered in the native HDL samples analyzed in the discovery study (Supplemental Table 3).

We presume that SM 42:3 corresponds to SM d18:2/24:1, which differs from typical sphingomyelins by the presence of an additional $\Delta^{14}$ cis-double bond in the sphingoid base backbone (Supplemental Figure 7A). We synthesized this atypical sphingadienine-based sphingomyelin (see Methods section), as well as the typical sphingosine-based SM d18:1/24:1 (SM 42:2) as the control lipid (Supplemental Figure 7). The analysis of the synthetic SM d18:2/24:1 and SM d18:1/24:1 by liquid chromatography-tandem MS (LC-MS/MS) reveals identical chromatographic retention times and fragmentation patterns as for the HDL-derived SM 42:3 and SM 42:2, respectively, confirming identity (Supplemental Figure 8). The size distribution of rHDL is not altered by the addition of either SM 42:3 or SM 42:2 (Supplemental Figure 9). Both the sphingadienine-based SM 42:3 and the sphingosine-based SM 42:2 increase the ability of rHDL

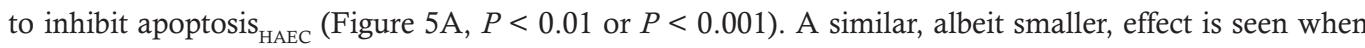
protein-free small unilamellar vesicles (SUVs) consisting of DOPC are used. In the absence of sphingomyelins, SUVs do not inhibit the apoptosis of HAECs. However, the incorporation of either SM 42:2 or SM 42:3 conveys some antiapoptotic activity to SUVs, which is lower than in rHDL but statistically significant (Figure 5B). Neither SM 42:3 nor SM 42:2 alters any other activity of rHDL (Supplemental Figure 10).

At no concentration did the incorporation of GPLD1 change the size distribution of rHDL (Supplemental Figure 9). The complementation of rHDL with GPLD1 improves the ability of rHDL to inhibit apoptosis $_{\text {HAEC }}$ (Figure 6A). The antiapoptotic effect of GPLD1 is encountered at all dosages (Figure 6A, $P<0.01)$. Of note, GPLD1 inhibits apoptosis of HAECs only if associated with rHDL but not as a free protein (Figure 6B). The antiapoptotic activity of rHDL containing GPLD1 is neutralized in the presence of an anti-GPLD1 antibody but not in the presence of a control IgG (Figure 6C). The addition of GPLD1 also increases the ability of rHDL to inhibit apoptosis ${ }_{\text {INS1e }}$ at the medium concentration (Supplemental Figure $11 \mathrm{~B}, P<0.01$ ), to reduce $\mathrm{MMP}_{\mathrm{C} 2 \mathrm{C} 12}$ at medium and high concentrations (Supplemental Figure 11D, $P$ $<0.001$ and $P<0.05$, respectively), and to promote respiration $\max _{\text {ax }}$ and respiration cAMP $_{\text {in brown adipocytes }}$ at low concentration (Supplemental Figure 11, $\mathrm{H}$ and I, both $P<0.05$ ).

The presence of apoF does not change the size distribution of rHDL (Supplemental Figure 9). ApoF increases the ability of $\mathrm{rHDL}$ to enhance respiration $\max _{\text {ax }}$ at medium concentration (Figure $7, P<0.05$ ). 
A

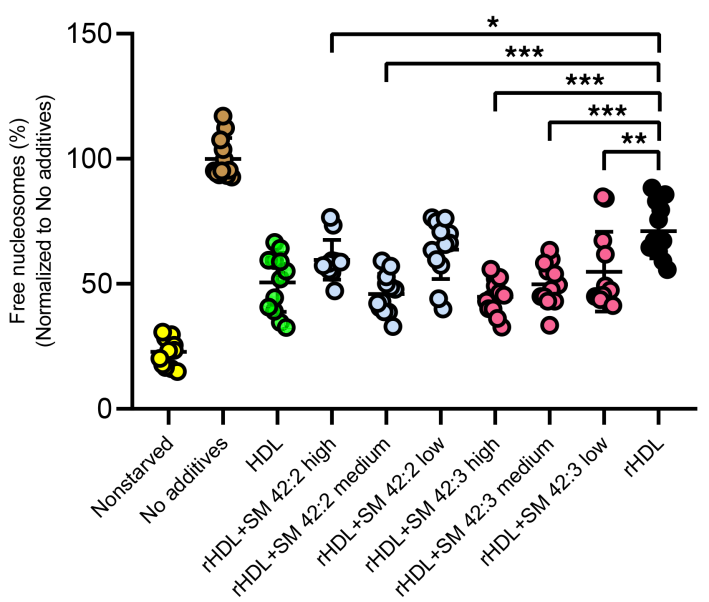

B

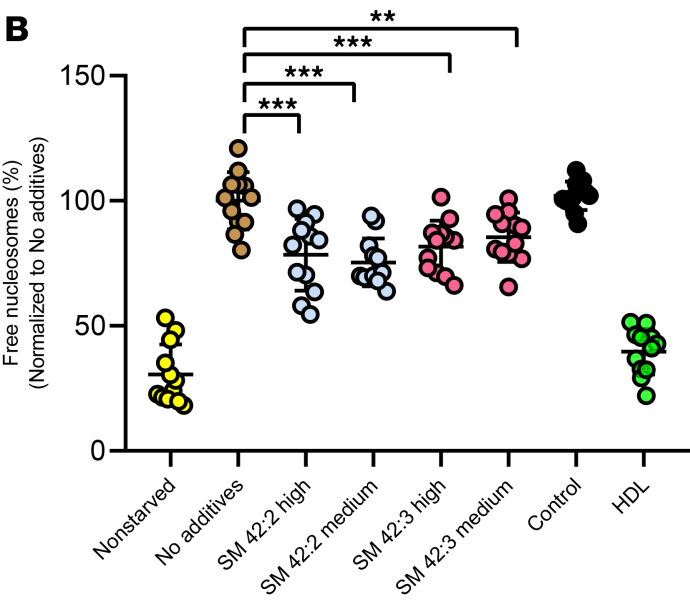

Figure 5. Sphingomyelins SM 42:2 and SM 42:3 decrease apoptosis in human aortic endothelial cells (HAECs). (A) Reconstituted HDL (rHDL) contain 3 different concentrations of SM 42:2 or SM 42:3, which correspond to the lowest, median, and highest concentration relative to phosphatidylcholine in native HDL. HAECs were starved in the absence or presence of $\pm 20 \mu \mathrm{g} / \mathrm{mL}$ rHDL for 16 hours. Apoptosis was recorded by using the free nucleosome assay. (B) SM 42:2 and SM 42:3 were reconstituted into unilamellar vesicles with 2 different concentrations. HAECs were starved in absence and presence of unilamellar vesicle \pm SM 42:2 or SM 42:3 for 16 hours, and apoptosis was recorded by free nucleosome assay. Data are presented as mean \pm SD of 3 independent experiments, each with 4 replicates, and were analyzed by 1-way ANOVA coupled with Dunnett's test for multiple comparisons against rHDL and no additives. ${ }^{* *} P<0.001 ;{ }^{* *} P<0.01 ;{ }^{*} P<0.05$.

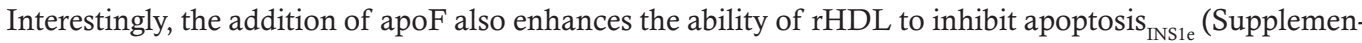
tal Figure 12B, $P<0.01$ at highest concentration) and to increase $\mathrm{MMP}_{\mathrm{C} 2 \mathrm{C} 12}$ (Supplemental Figure 12D, $P<0.05$ at highest concentration).

\section{Discussion}

Detailed knowledge of structure-function-disease relationships of HDL-associated molecules is a prerequisite to test them for their relative physiological and pathogenic importance and to exploit them for treatment and diagnostics. We therefore comprehensively and systematically characterized HDL particles of 51 healthy subjects and 98 patients with either CHD, T2DM, or both. Statistical modeling of 40 clinical characteristics, 34 NMR features, 182 proteins, 227 lipid species, and 12 functional read-outs resulted in the following 3 main findings. First, CHD and T2DM are associated with different changes of HDL in protein and lipid composition, as well as functionality. Second, different functions of HDL show rather little correlations with each other and are determined by different structural components. Third, we identified and verified 3 potentially novel structural determinants of HDL functions, namely apoF, GPLD1, and the sphingadienine-based sphingomyelin SM 42:3.

Associations of diseases with distinct structural and functional differences of HDL. Most previous studies compared HDL of healthy subjects with HDL of patients with 1 specific disease for structural differences in either size distribution, the proteome, or the lipidome, as well as 1 single function. Therefore, it is unclear whether alterations are disease specific and how they interact with each other. By our comprehensive systems biology approach, we found that, compared with $\mathrm{HDL}_{\text {heathy }}, \mathrm{HDL}_{\mathrm{CHD}}$ and $\mathrm{HDL}_{\mathrm{T} 2 \mathrm{DM}}$ share some but differ by most structural and functional alterations. The few similarities between $\mathrm{HDL}_{\mathrm{CHD}}$ and $\mathrm{HDL}_{\mathrm{T} 2 \mathrm{DM}}$ observed in the univariate volcano plot analysis (Supplemental Figures 1-3) disappeared upon multiple logistic regression with elastic net regularization (Figure 2).

NMR spectroscopy of plasma revealed the loss of large and very large HDL particles and the enrichment of small triglyceride-rich HDL in T2DM (Supplemental Figure 1). Using logistic regression with elastic net regularization, the loss of very large HDL particles emerged as a stable and independent feature of T2DM (Figure 2). Multivariate analysis based on PLS regression also unraveled the association of T2DM with the loss of very large HDL, but the association with a gain of small HDL was even stronger (Figure 3). These changes are probably caused by increased CETP activity (9-11). Opposite to T2DM, CHD is associated with the loss of small and medium-sized HDL and the gain of large HDL (Figure 3). Previous NMR studies also found more consistent and stable associations of HDL size distribution with T2DM rather than 
A

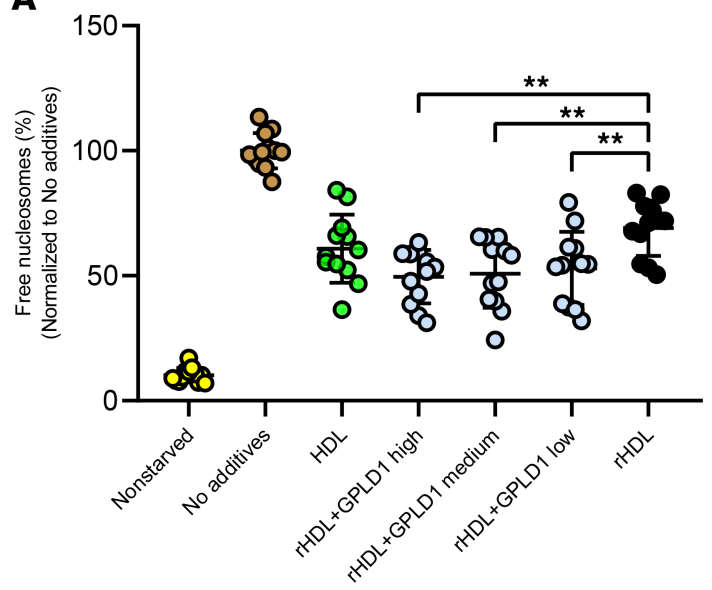

C

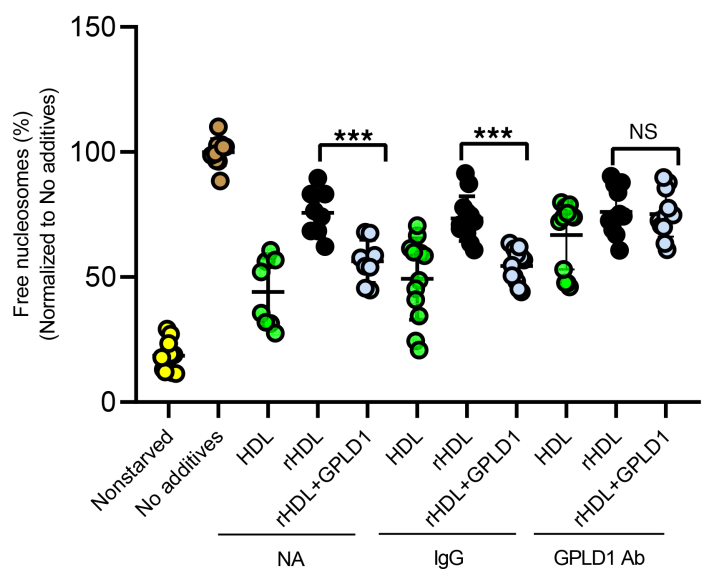

B

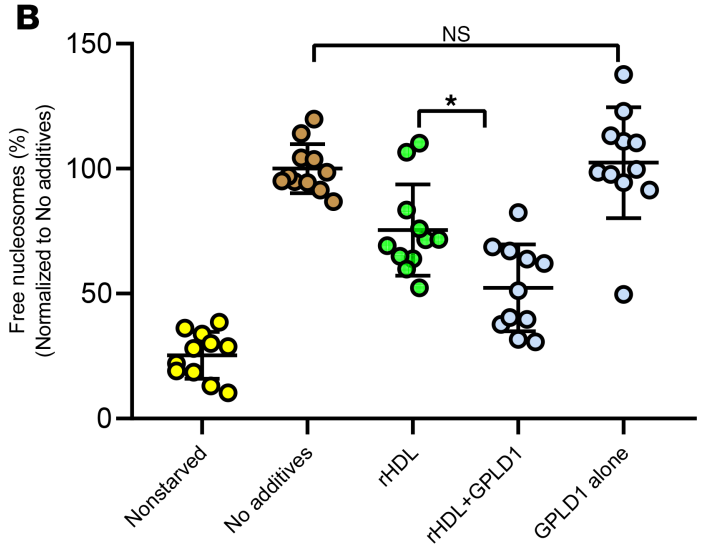

Figure 6. HDL-associated glycosylphosphatidyl-inositol specific phospholipase D1 (GPLD1) decreases apoptosis in human aortic endothelial cells (HAECs). (A) Reconstituted HDL (rHDL) were made with 3 different concentrations of GPLD1, reflecting the lowest, median, and highest concentration relative to apolipoprotein A-I (apoA-I) encountered in native HDL. HAECs were starved in the absence or presence of $20 \mu \mathrm{g} /$ $\mathrm{mL} r \mathrm{rHL}$. Data represent mean $\pm \mathrm{SD}$ of 3 independent experiments, each with 4 replicates, and were analyzed by 1-way ANOVA coupled with Dunnett's test for multiple comparisons against rHDL. (B) HAECs were starved in the absence or presence of $20 \mu \mathrm{g} / \mathrm{mL} \mathrm{rHDL} \pm$ GPLD1 or $200 \mathrm{ng} /$ $\mathrm{mL}$ free CPLD1 for 16 hours. (C) A total of $25 \mu \mathrm{g} / \mathrm{mL}$ native $\mathrm{HDL}$ or $\mathrm{rHDL} \pm$ GPLD1 were preincubated with $1 \mu \mathrm{g} / \mathrm{mL}$ IgG or anti-CPLD1 antibody. Cells were starved with $\mathrm{HDL}$ or $\mathrm{HHDL} \pm$ antibodies for 16 hours. Apoptosis was recorded by free nucleosome assay. (B and $\mathbf{C}$ ) Data represent mean \pm SD of 3 independent experiments, each with 4 replicates, and were analyzed by 1-way ANOVA coupled with Tukey's test for multiple comparisons. ${ }^{* *} P<0.001 ;{ }^{* *} P<0.01 ;{ }^{*} P<0.05$.

with CHD (12-17). Like in our study, both present and incident T2DM were previously associated positively with small HDL and inversely with larger $\operatorname{HDL}(14,15)$, whereas CHD was rather inversely associated with small and medium-sized HDL (12).

The lipidomic analysis of isolated HDL revealed many lipid species at reduced amounts in both $\mathrm{HDL}_{\mathrm{CHD}}$ and $\mathrm{HDL}_{\mathrm{T}_{2 \mathrm{DM}}}$, as compared with $\mathrm{HDL}_{\text {healthy }}$. Compared with $\mathrm{HDL}_{\mathrm{CHD}}$, a higher number of lipid species is altered in $\mathrm{HDL}_{\mathrm{T} 2 \mathrm{DM}}$ upon volcano plot analysis (Supplemental Figure 2), as well as upon the logistic regression and multivariate analyses (Figures 2 and 3). Relative to $\mathrm{HDL}_{\text {healthy }}$, both $\mathrm{HDL}_{\mathrm{T} 2 \mathrm{DM}}$ and $\mathrm{HDL}_{\mathrm{CHD}}$ are characterized by the enrichment of polyunsaturated PEs and the loss of PC and PI species that likely contain monounsaturated fatty acids (PC 36:2, PC 34:2, PI 36:2, and PI 34:2) (Supplemental Figure 2). However, upon logistic regression analysis, none of these associations remained statistically significant, except the association of PI 36:2 with $\mathrm{HDL}_{\text {СНD+T2DM }}$ (Figure 2). This analysis, however, revealed significant inverse associations of T2DM with lysophosphatidylcholines LPC 22:5 and, less strongly, LPC18:0. LPC18:0, LPC18:2, and LPC22:5 are also part of a cluster encompassing very large HDL, ether phosphatidylcholines, and sphingomyelins; the cluster is inversely associated with T2DM (Figure 3). Also of note are the strong positive associations of PE 38:5 and PE 40:7 with CHD upon multivariate analysis based on PLS regression (Figure 3). Ståhlman et al. also found LPC 18:1 and LPC 18:2 decreased in $\mathrm{HDL}_{\text {T2DM }}$ (18). The most prominent and independent inverse association of LPC 22:5 with T2DM (Figure 2) is of special note in view of the antiinflammatory effects of LPCs containing polyunsaturated fatty acids (19). Ether-PCs were previously reported to be decreased in $\mathrm{HDL}_{\mathrm{CHD}}(20)$ and in HDL of patients with metabolic syndrome (21), whereas others found them increased in $\mathrm{HDL}_{\mathrm{T} 2 \mathrm{DM}}$ (18). Sphingomyelins were reported to be decreased in HDL of patients with metabolic syndrome in 1 study but not in another $(18,22)$. An increased content of HDL in (polyunsaturated) PEs was previously reported for acute coronary syndrome patients and associated with increased thrombogenicity (23-25).

Compared with $\mathrm{HDL}_{\text {healthy }}, \mathrm{HDL}_{\mathrm{CHD}}$ was characterized by the gain of proteins, whereas $\mathrm{HDL}_{\mathrm{T} 2 \mathrm{DM}}$ rather showed a loss of proteins in the volcano-plot analysis (Supplemental Figure 3). Both $\mathrm{HDL}_{\mathrm{CHD}}$ and $\mathrm{HDL}_{\mathrm{T} 2 \mathrm{DM}}$ 
A

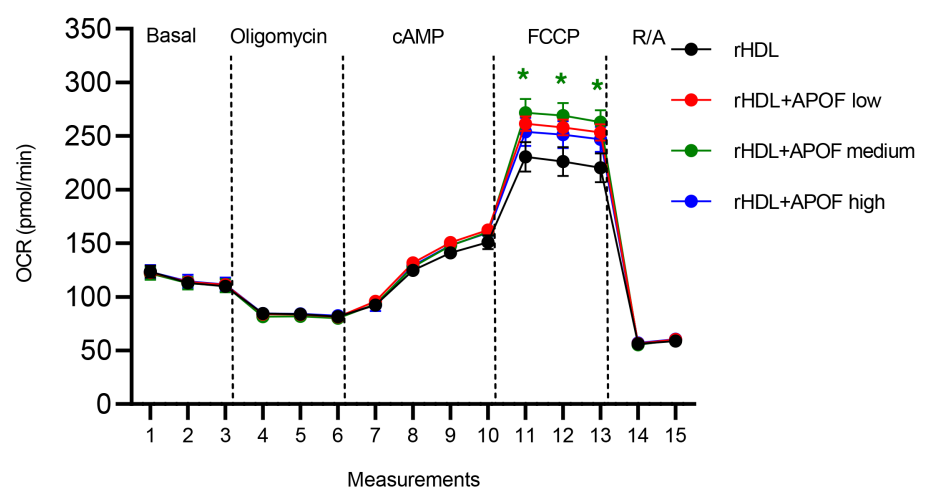

B

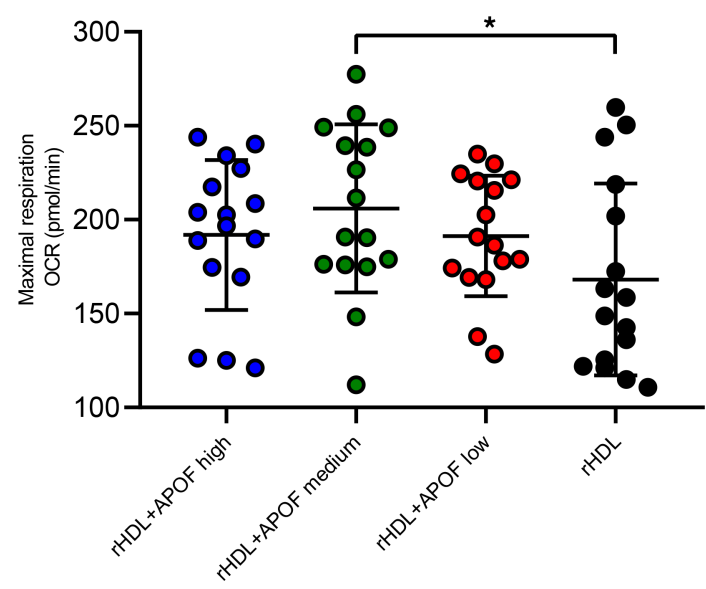

Figure 7. Apolipoprotein $\mathbf{F}$ (apoF) increases maximal respiration of human multipotent adipose-derived stem (hMADS) cells. Reconstituted HDL (rHDL) were prepared with 3 different concentrations of apoF, reflecting the lowest, medium, and highest concentration relative to apoA-I encountered in native HDL. (A and B) Oxygen consumption rate curves (A) and maximal mitochondrial respiration (B) in brown hMADS cells. Data represent mean \pm SD of 3 independent experiments, each with 4 replicates, and were analyzed by 1-way ANOVA coupled with Dunnett's test for multiple comparisons against rHDL. ${ }^{*} P<0.05$.

are deprived of apoA-IV but enriched with PSPB and SAAs (Supplemental Figure 3). The enrichment of $\mathrm{HDL}_{\mathrm{CHD}}$ with SAAs is known to interfere with HDL functions such as $\mathrm{CEC}$ and to be an adverse prognostic factor $(11,26,27)$. However, in our multiple regression analysis, no SAA protein showed any independent disease association. By contrast, the inverse association of apoA-IV with $\mathrm{CHD}$, as well as the positive association of PSPB with the combination of CHD and T2DM, is stably selected upon logistic regression analysis (Figure 2). Previous studies also reported an inverse association of apoA-IV with the presence and incidence of CHD (28-30). Interestingly, in a previous smaller study, we also found PSPB as the most significantly enriched protein of $\mathrm{HDL}_{\mathrm{CHD}}$ (31). Longitudinal studies found PSPB-enriched $\mathrm{HDL}$ as a significant predictor of mortality in T2DM patients undergoing hemodialysis, as well as in patients with heart failure $(32,33)$. We also found $\mathrm{HDL}_{\mathrm{T} 2 \mathrm{DM}+\mathrm{CHD}}$ and $\mathrm{HDL}_{\mathrm{T} 2 \mathrm{DM}}$ relatively deprived of apoE and apoD, respectively (Figure 2), which were also inversely associated with prevalent or incident diabetes by others (34). Interestingly, however, the PLS regression analysis unraveled the opposite association of apoD with the CHD cluster (Figure 3).

In agreement with the different structural changes, $\mathrm{HDL}_{\mathrm{CHD}}$ and $\mathrm{HDL}_{\text {T2DM }}$ are characterized by distinct dysfunctions (Supplemental Figure 4). Of note, CEC was not associated with any disease state, neither upon assessment with the most widely used bioassay of $\mathrm{HDL}$ function $\left(\mathrm{CEC}_{\text {apoB-free plasma }}\right)(8,35)$ nor upon recording of $\mathrm{CEC}_{\mathrm{HDL}}$ with isolated $\mathrm{HDL}$. Several less-frequently investigated functions are specifically altered in either $\mathrm{HDL}_{\mathrm{CHD}}$ or $\mathrm{HDL}_{\mathrm{T} 2 \mathrm{DM}} ; \mathrm{HDL}_{\mathrm{T} 2 \mathrm{DM}}$ is characterized by reduced anti-apoptotic activity toward HAECs and reduced ability to lower the MMP of $\mathrm{C} 2 \mathrm{C} 12$ myotubes. The latter association with T2DM remained stable upon logistic regression with elastic net regulation and stability selection (Figure 2), as well as upon multivariate analysis based on PLS regression (Figure 3), where T2DM, MMP $\mathrm{C} 2 \mathrm{C} 12_{2}$, and apoptosis $_{\mathrm{HAEC}}$ are clustered. Our initial data analyses found the ability of HDL to promote respiration max $_{\text {ax }}$ of brown adipocytes associated positively with CHD but inversely with T2DM (Supplemental Figure 4). Upon advanced statistical modeling, only the positive association of respiration ${ }_{\text {max }}$ with $\mathrm{CHD}$ persisted (Figures 2 and 3). In 2 previous studies, we found reduced antiapoptotic activity of $\mathrm{HDL}_{\mathrm{CHD}}$ toward $\operatorname{HAECs}(20,31)$, which we do not replicate in the present study. In those 2 previous studies, $\mathrm{CHD}$ encompassed both diabetic and nondiabetic subjects so that we cannot exclude confounding of CHD with T2DM as the reason for our previous finding of reduced antiapoptotic activity of $\mathrm{HDL}_{\mathrm{CHD}}$.

Structure-function relationships of $H D L$. In general, only related functions in the same cell systems, such as $\mathrm{CEC}_{\mathrm{HDL}}$ and $\mathrm{CEC}_{\text {apob-free plasma }}$, or $\mathrm{MMP}_{\mathrm{C2C12}}$ at different time points showed significant correlations (Supplemental Figure 5) and share the same or similar structural determinants (Figure 4). Distinct functions of HDL in different cell models are weakly correlated with one another, if at all (Supplemental Figure 5). In line with this, they share almost no common structural determinant (Figure 4 and Supplemental Figure 6). This divergence is of special note for CEC from both a biological and a clinical perspective. HDLs elicit many cellular responses, which have been explained by 2 principal paradigms. One paradigm interprets HDL-induced cellular functions 
as the secondary result of cholesterol efflux and, hence, altered cellular cholesterol homeostasis, which in turn results in transcriptional responses of sterol sensitive genes or alters the localization and activity of signaling receptors in raft domains of the plasma membrane $(3,7,36)$. The other paradigm assumes direct and specific interactions between HDL-bound agonists and cellular receptors $(3,7,37)$. The weakness of the correlations (Figure 2 and Supplemental Figure 5) and the differences in structural determinants (Figure 4 and Supplemental Figure 6) observed by us suggest that HDL elicits many actions independently of or in addition to its cholesterol efflux-stimulating activities. In this regard, it is noteworthy that we found CEC to be mainly determined by rather unspecific measures, such as HDL-C, apoA-I, small and medium-sized HDL, and different phosphatidylcholine species (Figure 4). Previous studies also found apoA-I and phosphatidylcholines, as well as medium-sized HDL, as the major determinants of $\operatorname{CEC}(8,21,35,38)$. Likewise, Lusis and colleagues found apoA-I and medium-sized HDL, rather than any minor HDL proteins, to be the most important explanatory factors of differences in $\mathrm{CEC}_{\mathrm{HDL}}$ from different mouse strains (39). Also in line with these and our findings, a recent genomewide association study found $\mathrm{CEC}_{\mathrm{apoB} \text {-free plasma }}$ associated with variants in genes encoding proteins involved in the formation and metabolism of HDL rather than for minor and nonclassical protein components of HDL (40). Beyond classical genes of HDL metabolism such as the APOLP1 and APOLP2 loci, CETP, LIPC, or LPL, 2 loci including the PLB1 and ENPP7 genes - which encode for enzymes degrading phosphatidylcholines and sphingomyelins - were the only ones associated with $\mathrm{CEC}_{\text {apoB-free plasma }}$ (40). Thus, general physicochemical properties such as number, size, and fluidity of particles rather than specific components appear to determine $\mathrm{CEC}_{\mathrm{HDL}}$. By contrast, we found other functions determined by specific quantitatively minor HDL components such as apoC-III and S100A9 (mitochondrial potential of myotubes), apoF and CATD (respiration of brown adipocytes), apoL1 (ER stress-induced apoptosis of INS1e cells), or GPLD1 (starvation-induced apoptosis of HAECs), which had no impact on $\mathrm{CEC}_{\mathrm{HDL}}$, neither in the bioinformatics approach (Figure 4) nor in the validation experiments (Supplemental Figures 11 and 12).

From a clinical point of view, the weak correlations of $\mathrm{CEC}_{\mathrm{HDL}}$ or $\mathrm{CEC}_{\text {apoB-free serum }}$ with other functions as well as their different structural determinants question the postulated potential of CEC as an integrative proxy of HDL functionality $(35,41)$. In several but not all studies, $\mathrm{CEC}_{\text {apoB-free plasma }}$ was found associated with the presence and incidence of CHD, independently of and more strongly than HDL cholesterol (35, 41). Moreover, although CETP inhibitor treatment was found to increase CEC (42), this intervention failed to prevent CHD events (5). Of note and in accordance with their clinical futility, treatment with CETP inhibitors failed to improve endothelial functionalities of HDL (43).

Determinants of HDL functions. The combination of statistical modeling and experimental validation led to the identification of 3 determinants of HDL function, namely the sphingadienine-based sphingomyelin SM 42:3 and GPLD1 for the ability of HDL to inhibit starvation-induced apoptosis of endothelial cells, as well as apoF for the ability of HDL to promote respiration ${ }_{\max }$ of brown adipocytes. These 3 molecules are poorly investigated.

Polyunsaturated sphingomyelins SM 36:3, SM 38:3, SM 42:3, and SM 42:4 emerged as determinants of HDL's activities to inhibit starvation-induced apoptosis of HAECs and lower the mitochondrial potential of C2C12 myotubes (Figure 4 and Supplemental Figure 6). Supplementation with SM 42:3 improved the antiapoptotic activity of both rHDL and SUVs (Figure 5). The backbone of SM 42:3 (and probably also the other polyunsaturated SMs) differs from that of typical sphingomyelins by the presence of sphingadienine, which contains 2 double bonds instead of sphingosine, which has only 1 double bond. The $\Delta^{14}$ cis-double bond generates an atypical nicked rather than the typical straight backbone of sphingoid bases (Supplemental Figures 7 and 8). The sphingadienine-based sphingolipids have been little investigated, although they have been identified already in the 1960 s and constitute $15 \%-20 \%$ of all plasma sphingolipids (44-46). We previously found plasma levels of sphingadienine inversely associated with the risk of major cardiovascular events during a follow-up of 8 years in patients who underwent coronary angiography (44). Most recently, plasma levels of sphingadienine-based lipids were found inversely associated with BMI and the HOMA insulin resistance index (47). Sphingadienine is likely generated by the desaturation of sphingosine through an as yet unknown enzyme. It is probably metabolized by phosphorylation-like sphinganines and sphingosines, since cells and plasma contain sphingadienine-phosphate (S1P d18:2) (20). S1P d18:2 probably acts like sphingosine-1-phosphate (S1P d18:1) as an agonist of sphingosine-1-phosphate receptors (48). In support of this, the contents of HDL in S1P d18:1 and S1P d18:2 show similar correlations with the antiapoptotic activity of HDL (20). In this regard, it is noteworthy that the sphingosine-based SM 42:2 also improved the ability of both rHDL and SUVs to inhibit the apoptosis of HAECs (Figure 5), as well as the ability of rHDL to lower MMP of myotubes (Supplemental Figure 10D). SM 42:3, but also other sphingomyelins, transported by HDL may serve as 
substrates for plasma membrane-located sphingomyelinase, ceramidase, and sphingosine-kinase, which — in serial reactions - generate S1P d18:1and S1P d18:2 (48). As agonists of S1P receptors, they would interfere with apoptosis of HAECs (49). Unfortunately, our lipidomic method did not record S1Ps.

GPLD1, also called GPI-PLD and encoded by the PHLD gene, is mainly produced by the liver. In plasma, about $90 \%$ of GPLD1 is recovered with apoA-I- or apoA-II-containing lipoproteinslipoproteins (i.e., HDL) (50). GPLD1 specifically hydrolyzes the glycan-phosphatidylinositol linkages of GPI-anchored proteins, which include essential membrane proteins such as receptors, protease inhibitors, and transporters. GPLD1 is assumed to play an important regulatory role for the activity of these proteins. However, little is known on the specific relevance of circulating GPLD1. GPLD1 improves the activity of rHDL to inhibit the apoptosis of HAECs (Figure 6A). Conversely, the antiapoptotic activity of both native HDL and GPLD1 containing rHDL was lowered by a neutralizing anti-GPLD1 antibody (Figure 6C). Of note, GPLD1 was not able to inhibit apoptosis in the absence of $\mathrm{HDL}$ (Figure 6B). Although not foreseen by the statistical modeling, $\mathrm{rHDL}$ increased $\mathrm{MMP}_{\mathrm{C} 2 \mathrm{C} 12}$ as well as respiration cAMP and respiration $\max$, in the presence of GPLD1. These effects - as well as the associations of T2DM with the GPLD1 content of HDL, apoptosis ${ }_{\mathrm{HAEC}}, \mathrm{MMP}_{\mathrm{C} 2 \mathrm{C} 12}$, and respiration max $_{\text {(Supplemental }}$ Figures 3 and 4) - point to a role of GPLD1 in diabetes. However, data from studies in genetic mouse models and humans are controversial. On the one hand, overexpression of hepatic GPLD1 was found to improve oral glucose tolerance and to reduce serum triglyceride catabolism in mice $(51,52)$. On the other hand, the $\mathrm{KO}$ of Phld in mice ameliorated glucose intolerance and hepatic steatosis under high-fat and high-sucrose diet (53). Cross-sectional studies found increased plasma levels of GPLD1 in subjects with prediabetes compared with normoglycemic subjects (54) and in patients with diabetes mellitus type 1 compared with both normoglycemic subjects and T2DM patients (55). To solve these controversies, it will be important to unravel the mode of action of GPLD1 in HDL, notably to identify the GPI-anchored proteins that are shed by GPLD1 and modulate apoptosis, as well as mitochondrial potential and respiration.

Finally, we identified apoF as a determinant of HDL's ability to promote respiration ${ }_{\max }$ in brown adipocytes (Figures 4 and 7). Although not foreseen by the statistical modeling, apoF also improved the ability of rHDL to inhibit the ER stress-induced apoptosis of INS1e cells (Supplemental Figure 12B). ApoF was previously discovered as an endogenous CETP inhibitor (56). Our findings on specific effects of apoF on mitochondrial respiration of brown adipocytes and ER stress-induced apoptosis of INS1e cells point to specific activities of apoF, which are independent of CETP inhibition. In agreement with this, the overexpression of apoF in mice, which lack CETP, decreased HDL cholesterol (57). The KO of Apof had no effect on concentrations and size distribution of HDL and other plasma lipoproteins (58), which is in agreement with the size distribution of rHDL being unaltered by the addition of apoF (Supplemental Figure 7). Also in agreement with our statistical analysis and in vitro experimental data, $\mathrm{CEC}_{\text {apoB-free plasma }}$ was not altered by the addition of apoF (58). In hamsters, which - in contrast to mice - express CETP, RNA interference with apoF had no effect on lipoprotein distribution upon chow diet but increased LDL cholesterol and decreased HDL cholesterol upon high-fat feeding (59). Interestingly, the additional $\mathrm{KO}$ of Apof decreased atherosclerosis in $L d l r$-KO mice. This effect, however, could not be causally related to the lack of apoF because the Apof KO was associated with hypomorphic expression of the closely linked Stat2 gene (60). The previously reported inverse correlation between plasma levels of apoF and triglycerides $(61,62)$ is in agreement with our finding of low apoF levels in $\mathrm{HDL}_{\mathrm{T} 2 \mathrm{DM}}$ and $\mathrm{HDL}_{\mathrm{T} 2 \mathrm{DM}+\mathrm{CHD}}$ (Supplemental Figure 3).

Limitations and conclusions. Our study has several limitations. First, although - to our knowledge - our study is the largest and most comprehensive systems biology study on HDL, the sample number of our study is rather small. Moreover, due to the high prevalence of asymptomatic atherosclerosis, we cannot rule out that some of our healthy control subjects had CHD. Thus the associations of specific lipids and proteins with T2DM or CHD and, hence, their utility as prognostic biomarkers needs to be validated in targeted longitudinal clinical or epidemiological studies. Second, our findings on specific disease associations of HDL components do not imply any direct or reverse causal relationships. This caveat is not only generally valid, but also specifically relevant for our study of patients and control subjects who were not matched by potential confounders. However, our primary aim was to test the hypothesis that T2DM, CHD, and the combination thereof cause general alterations of HDL structure and function. This hypothesis is falsified by our findings. Any causal relationship between the disease status and the alterations in the structural composition, structure, and functions of HDL, as well as their directionality, needs to be investigated in targeted experiments and genetic studies. Third, the weakness or lack of correlation between different functions and the finding of different structural determinants may be biased by the analysis of different functions in different cell culture systems. 
Indeed, different agonists may be active in different cell system - for example, depending on the expression of receptors. However, the claim of a uniform structure-function relationship of HDL is driving the use of $\mathrm{CEC}$ assays in clinical studies. Our findings clearly rule out that $\mathrm{CEC}_{\text {apoB-free plasma }}$ or $\mathrm{CEC}_{\mathrm{HDL}}$ are proxies for all other cellular HDL functions. Forth, for most studies, we used transformed and nonhuman cell lines for the identification and verification of agonists or determinants of HDL function. Whether or not they are relevant for primary cells and even more so in vivo needs to be investigated by follow-up studies. Further work is also needed to identify the mode of action by which GPLD1, apoF, and SM 42:3 exert HDL functions.

\section{Methods}

Volunteers and patients. Fifty-one healthy volunteers, 46 patients with T2DM only, and 19 patients with both T2DM and coronary heart disease (CHD) were recruited at the University Hospital Zurich. Twenty-five patients with CHD only and 8 patients with both CHD and T2DM were recruited at Charité Universitätsmedizin Berlin. As the exclusion criteria, we defined pregnancy, presence of any acute disease or decompensation of chronic disease (except acute coronary syndrome), severe chronic kidney disease (eGFR $<30$ $\mathrm{mL} / \mathrm{min}$ ), and active inflammation (C-reactive protein $>10 \mathrm{mg} / \mathrm{L}$ or leukocytes $>10 \mathrm{~g} / \mathrm{L}$ ). Data from 2 patients were omitted due to lacking lipidomic measurements; hence, we report on 149 probands with no missing data for HDL function or structure.

A pool plasma was prepared from 3 blood donors to be used as the precision control.

Clinical chemistry and NMR spectroscopy of plasma. Activities of liver enzymes, as well as concentrations of total and HDL cholesterol, triglycerides, glucose, creatinine, and CRP, were measured by the use of a COBAS8000 analyzer and assays from Roche Diagnostics. LDL cholesterol was calculated by the Friedewald formula. Concentrations of apoA-I and apoB were measured by immunonephelometry using the BN Prospec and assay from Siemens Healthcare Diagnostics. Lipoprotein(a) (Lp[a]) was measured by the use of a latex enhanced immunoturbidimetric assay from Randox Laboratories Ltd. Glycated hemoglobin (HbA1c) was measured by HPLC (ADAMS A1c, ARKRAY).

NMR spectroscopy of lipoproteins was performed by Nightingale Health (http://nightingalehealth.com).

Isolation of HDL. HDL were isolated by 14 ultracentrifuge runs, each of which included 11 experimental samples and 1 pool plasma for internal quality control. Two ultracentrifuges were run in parallel, yielding 4 batches of HDL each from 22 study samples and 2 quality controls. Each batch was composed of 5 or 6 samples from each study group. Each HDL isolate was diluted to yield the same concentration $(2 \mathrm{mg} / \mathrm{mL})$ and then divided into appropriate aliquots for each lab. The aliquots were encoded so that the analyzing personnel was blind for their patient group assignment. Batches of 22 samples plus controls were maintained at $4^{\circ} \mathrm{C}$ for maximally 3 days until the bioassays were performed. Aliquots for proteomics and lipidomics were frozen immediately at $-80^{\circ} \mathrm{C}$ until the analyses were performed in 2 batches of 88 samples plus 8 quality control pools and 63 samples plus 6 quality controls.

For the calibration of the bioassays, we used rHDL (CSL111) provided by CSL Behring.

Preparation of reconstituted rHDL and SUVS. For functional validation of our candidate lipids and proteins, we reconstituted $\mathrm{rHDL}$ by the use of the cholate dialysis method $(20,31)$. rHDL consisted of apoA-I and DOPC with or without the protein or lipid of interest. The respective candidate proteins or lipids were included at 3 different amounts, reflecting the lowest, median, and highest concentration measured in the native HDL samples (Supplemental Table 3). ApoA-I was purified as reported previously (63). DOPC, ApoF, and GPLD1 were purchased from Avanti Polar Lipids, Aviva Systems Biology, and OriGene Technologies, respectively. For neutralization of GPLD1, $25 \mu \mathrm{g} / \mathrm{mL}$ native HDL or $\mathrm{rHDL} \pm$ GPLD1 were preincubated with $1 \mu \mathrm{g} / \mathrm{mL}$ antiGPLD1 antibody (ab192543, Abcam) or control IgG (ab176094, Abcam) for 1 hour. SM 42:3 and SM 42:2 were synthesized as described below. The size distribution of the HDL particles was analyzed by nondenaturing polyacrylamide gel electrophoresis. SUVs consisted of DOPC and the respective sphingomyelin in molar ratios of 66.6:33.3 (SM 42:2 high or SM 42:3 high), 90:10 (SM 42:2 medium or SM 42:3 medium) or 100:0 (control). The lipid suspension was passed 30 times through a membrane (pore size $200 \mathrm{~nm}$ ). Thereafter, residual large particles were removed by centrifugation at 20,000 $\mathrm{g}$ for 15 minutes to yield a clear suspension of SUV.

Synthesis of sphingomyelins SM42:3 and SM42:2. The synthesis of SM 42:2 (SM d18:1/24:1) and SM 42:3 (SM d18:2/24:1) is depicted in Supplemental Figure 7A. Details are described in Supplemental Methods 1. The identity and purity of SM 42:3 (SM d18:2/24:1) and SM 42:2 (SM d18:1/24:1) were analyzed by NMR spectroscopy (not shown). The identity of synthetic SM d18:2/24:1 and SM d18:1/24:1 with SM 42:3 and SM 42:2 in HDL was assessed by LC-MS/MS (Supplemental Figure 8). 
Proteomics. HDL was delipidated using an extraction protocol by Wessel and Flügge (64). To control for extraction efficiency and reproducibility, bovine $\alpha$-1-Acid-Glycoprotein (A1AG) was spiked into each sample as protein reference prior to digestion. Proteins isolated from HDL underwent tryptic digestion and were subsequently analyzed on a QExactive HF Hybrid Quadrupole-Orbitrap Mass Spectrometer (Thermo Fisher Scientific) using data-dependent (and data-independent) acquisition schemes (DDA and DIA, respectively). Details are described in Supplemental Methods 2.

Lipidomics. Lipids were quantified by direct flow injection electrospray ionization-tandem MS (ESI$\mathrm{MS} / \mathrm{MS})$ in positive ion mode using the analytical setup and strategy described previously $(65,66)$. Details are described in Supplemental Methods 3.

Bioassays. The characteristics of bioassays are summarized in Supplemental Table 2.

$\mathrm{CEC}$ of both isolated HDL $\left(\mathrm{CEC}_{\mathrm{HDL}}\right)$ and apoB-free plasma $\left(\mathrm{CEC}_{\text {apoB-free plasma }}\right)$ was measured as reported by Ritsch et al. (67). In brief, J774 (ATCC, TIB-67) macrophages were radiolabeled with $2.5 \mu \mathrm{Ci} / \mathrm{mL}$ ${ }^{3} \mathrm{H}$-cholesterol and stimulated with $0.2 \mathrm{mM}$ cAMP (MilliporeSigma) to upregulate ABCA1. The efflux medium containing the stimuli apoB-depleted plasma or HDL was added to the cells for 4 hours. All steps were performed in the presence of $5 \mu \mathrm{g} / \mathrm{mL}$ acyl-coenzyme A/cholesterol acyltransferase inhibitor (Sandoz).

To record the antiapoptotic activity of HDL toward HAECs (Cell Applications Inc., 304-05a), 6 passages old, and confluently grown HAECs were starved overnight and then incubated with $25 \mu \mathrm{g} / \mathrm{mL}$ native HDL or $10 \mu \mathrm{g} / \mathrm{mL}$ CSL111 or $20 \mu \mathrm{g} / \mathrm{mL} \mathrm{rHDL}$. Apoptosis ${ }_{\mathrm{HAEC}}$ was recorded by the use of an ELISA from Roche Diagnostics that monitors the amount of nucleosomes in the cytoplasmic fraction of cell lysates, which occurs in the later phase of apoptosis.

To record the ability of HDL to protect $\beta$ cells from ER stress-induced apoptosis (Apoptosis INS1e $_{1}$ ), 62-passages-old and confluently grown cells of the rat pancreatic $\beta$ cell line INS1e (provided by C. Wollheim, Geneva; ref. 68) were stimulated with $100 \mathrm{nM}$ Thapsigargin (Sigma Aldrich, catalog T9033) and incubated with $75 \mu \mathrm{g} / \mathrm{mL}$ native HDL, $10 \mu \mathrm{g} / \mathrm{mL}$ CSL111, or $10 \mu \mathrm{g} / \mathrm{mL}$ rHDL. Apoptosis ${ }_{\text {INS1e }}$ was recorded by the use of the Cell Death ELISA from Roche Diagnostics.

Mitochondrial integrity is of paramount importance to cellular health since cells with reduced mitochondrial function initiates cytochrome c-dependent apoptosis. Elevated mitochondrial membrane potential (MMP) is associated with increased ROS production and therefore is deleterious. To understand the effect of HDL on MMP, C2C12 myotubes (ATCC, CRL-1772) were differentiated in vitro for 4 days and serum fasted overnight; they were then subjected to serum starvation for 2 hours the following day and incubated with $100 \mathrm{ng} / \mathrm{mL}$ of HDL for an additional 2 hours. MMP was monitored using the JC-1 fluorescent dye (Abcam, catalog ab141387) that accumulates in mitochondria in a voltage-dependent manner (dye was loaded for 30 minutes prior to serum starvation). HDL protects against the increase in MMP associated with serum starvation in a dose-dependent manner. The experiment was terminated by the addition of CCCP, a protonophore that dissipates the potential across the inner mitochondrial membrane, to ensure the reliability of JC-1 as a read-out of MMP.

To study the effect of HDL derived from healthy and diseased patients on brown adipocyte activity, we employed human multipotent adipose-derived stem cells (hMADS) generated and provided by Ez-Zoubir Amri from CNRS (Institute of Biology Valrose, Nice, France); hMADS constitute a unique human cell model that can be differentiated into functional brown adipocytes (69). Cells were grown and differentiated into mature white adipocytes on 96-well collagen-coated Seahorse plates. Brown adipocytes were obtained by an additional rosiglitazone (Adipogen, catalog 71740; $100 \mathrm{nM}$ ) treatment from day 14-17. Mature brown adipocytes were exposed to $100 \mu \mathrm{g} / \mathrm{mL}$ of HDL, rHDL, CSL111, or PBS as control for 3 hours before measurement of mitochondrial respiration using the Seahorse 96XF mito-stress test kit. After measurement of the basal respiration, oligomycin (Adipogen, catalog 11342;1 $\mu \mathrm{g} / \mathrm{mL}$ inhibitor of complex V; Adipogen, catalog11342) was injected to block respiration coupled to ATP synthesis. Decrease in oxygen consumption rate (OCR) following oligomycin injection reflects contribution of coupled respiration to the basal mitochondrial OCR. Uncoupled respiration was, in the next step, induced with cAMP (0.5 mM dibutyryl cAMP; Sigma-Aldrich, catalog D0627) to quantify the capacity of cells to dissipate energy through uncoupled respiration. FCCP (Sigma-Aldrich, catalog C2920; $1 \mu \mathrm{g} / \mathrm{mL}$ ), a chemical uncoupler, was injected to fully uncouple the mitochondrial membrane and to quantify the maximal respiratory capacity of brown adipocytes. In the last step, rotenone (Sigma-Aldrich, catalog R8875; $3 \mu \mathrm{M}$ ) and antimycin A (Sigma-Aldrich, catalog A8674; $2 \mu \mathrm{g} / \mathrm{mL}$ ) were injected to block mitochondrial respiration (complex I and III) and estimate contribution of nonmitochondrial respiration to the measured OCR. Nonmitochondrial respiration was subtracted to obtain basal, basal uncoupled, cAMP-stimulated uncoupled, and maximal 
mitochondrial respiration. Using this protocol, we could reliably quantify the effect of HDL derived from healthy and diseased patients on individual parameters of mitochondrial respiration.

Statistics. The details of all statistical methods are provided in Supplemental Methods 4 . In the clinical data, approximately $1 \%$ of values were missing and were imputed by their predicted values from linear regression models fit on all clinical covariates (70). We logarithmically transformed the MS and NMR spectroscopy data after adding an estimate of the lower limit of quantification for each feature (71). In order to adjust for nuisance variables, we linearly regressed each protein, lipid, or lipoprotein particle feature on indicator variables of diabetes, CHD, hospital, sex, and - when applicable - HDL isolation date and centrifuge. Effects of nuisance variables were removed, and disease effects were retained for downstream analysis. We used volcano plots to visualize univariate associations between disease conditions and abundance of proteins, lipid species, and subclasses of HDL. The resulting $P$ values were adjusted for multiple comparisons using the Benjamini-Hochberg procedure (72) for controlling the FDR. For the functional bioassays, we used linear mixed-effects models (73) to adjust for nuisance variables and plate effects while accommodating technical replicates of HDL samples.

We used logistic regression models to estimate associations between disease conditions and clinical, functional, and structural data. Since the data contained fewer observations than features, and due to the considerable correlation among the predictors, we used elastic net regularization (74) in order to make the regression problem identifiable. The elastic net parameters were tuned simultaneously using bivariate cross-validation. In addition, we used stability selection $(75,76)$ in order to bound the expected number of falsely selected variables.

In order to leverage the mutual information between disease conditions and HDL functions, we modeled them as a multivariate response vector using PLS regression (77) in a sparse formulation (78) and tuned the model parameters through cross-validation.

With the aim of finding candidate relationships between HDL function and structure, we used a Gaussian graphical model to infer conditional dependencies between continuous-valued features. This amounted to estimating partial correlations, which reflect the dependence between each pair of variables conditioned on all other variables in the model, thereby adjusting for the influence of observed confounders (79). Due to the high dimensionality, we regularized the model using the graphical lasso (80). Since the distributions of our features typically had higher kurtosis (heavier tails) than a normal distribution, we used the nonparanormal formulation (81) together with a stability approach to tune regularization (82).

Study approval. Ethics approval was obtained from Kantonale Ethikkommission Zurich (PB-BASEC_2015-00159) and Ethikkommission der Charité at Universitätsmedizin Berlin (EA4/123/16). All volunteers and patients participated with informed consent.

\section{Author contributions}

AVE, BW, CW, JK, M. Claassen, and NB designed the study and obtained funding. AVE, JH, JK, NK, and UL obtained ethical approval for the study and recruited patients and healthy volunteers. LR, MY, and SR managed the biobank, isolated HDL, and prepared rHDL. AS and EC synthesized SM 42:3 and SM 42:2. AR, EL, LR, MB, MH, MK, MY, GK, and SR performed or supervised experiments. SG performed and BW supervised proteomics. AO and GL performed lipidomics. M. Cardner performed and NB supervised the statistical analyses and modeling. AO, AR, AVE, BW, CW, EL, JK, LR, MB, M. Cardner, MH, MY, $\mathrm{NB}$, SG, and TH analyzed and interpreted data. AVE wrote the first draft of the manuscript. All authors critically read and revised the manuscript.

\section{Acknowledgments}

This work was supported by Systems X Program Grant MRD 2014/267 to AVE, BW, CW, JK, MC, and NB; a Sinergia grant from the Swiss National Science Foundation (SNF, CRSII3_154420) to AVE; and funds from the Austrian Science Foundation (FWF), Vienna, Austria (P27116-B23) to AR. We thank the staff of the Clinical Trial Center for the recruitment and management of healthy volunteers and patients, Doreen Müller for excellent technical assistance in the lipidomic analysis, and Giusi Moffa as well as Jack Kuipers for useful discussions about the statistical analysis. We thank Samuel Wright (CSL Behring) for providing CSL111 used for the calibration of bioassays.

Address correspondence to: Arnold von Eckardstein, Institute of Clinical Chemistry, University Hospital of Zurich, Rämistrasse 100, 8091 Zurich, Switzerland. Phone: 41.44.255.2260; Email: arnold. voneckardstein@usz.ch. 
1. Holmes MV, Ala-Korpela M, Smith GD. Mendelian randomization in cardiometabolic disease: challenges in evaluating causality. Nat Rev Cardiol. 2017;14(10):577-590.

2. März W, et al. HDL cholesterol: reappraisal of its clinical relevance. Clin Res Cardiol. 2017;106(9):663-675.

3. Annema W, von Eckardstein A. High-density lipoproteins. Multifunctional but vulnerable protections from atherosclerosis. Circ $J$. 2013;77(10):2432-2448

4. von Eckardstein A, Widmann C. High-density lipoprotein, beta cells, and diabetes . Cardiovasc Res. 2014;103(3):384-394

5. Tall AR, Rader DJ. Trials and Tribulations of CETP Inhibitors. Circ Res. 2018;122(1):106-112.

6. Ference BA, et al. Low-density lipoproteins cause atherosclerotic cardiovascular disease. 1. Evidence from genetic, epidemiologic, and clinical studies. A consensus statement from the European Atherosclerosis Society Consensus Panel. Eur Heart J. 2017;38(32):2459-2472.

7. Annema W, von Eckardstein A. Dysfunctional high-density lipoproteins in coronary heart disease: implications for diagnostics and therapy. Transl Res. 2016;173:30-57.

8. Talbot CPJ, Plat J, Ritsch A, Mensink RP. Determinants of cholesterol efflux capacity in humans. Prog Lipid Res. 2018;69:21-32.

9. Blauw LL, et al. Mendelian randomization reveals unexpected effects of CETP on the lipoprotein profile. Eur J Hum Genet. 2019;27(3):422-431.

10. Gomez Rosso L, et al. Poor glycemic control in type 2 diabetes enhances functional and compositional alterations of small, dense HDL3c. Biochim Biophys Acta Mol Cell Biol Lipids. 2017;1862(2):188-195.

11. Rached F, et al. Defective functionality of small, dense HDL3 subpopulations in ST segment elevation myocardial infarction: Relevance of enrichment in lysophosphatidylcholine, phosphatidic acid and serum amyloid A. Biochim Biophys Acta. 2015;1851(9):1254-1261.

12. Ditah C, Otvos J, Nassar H, Shaham D, Sinnreich R, Kark JD. Small and medium sized HDL particles are protectively associated with coronary calcification in a cross-sectional population-based sample. Atherosclerosis. 2016;251:124-131.

13. Arsenault BJ, et al. Comparison between gradient gel electrophoresis and nuclear magnetic resonance spectroscopy in estimating coronary heart disease risk associated with LDL and HDL particle size. Clin Chem. 2010;56(5):789-798.

14. Mora S, Otvos JD, Rosenson RS, Pradhan A, Buring JE, Ridker PM. Lipoprotein particle size and concentration by nuclear magnetic resonance and incident type 2 diabetes in women. Diabetes. 2010;59(5):1153-1160.

15. Garvey WT, et al. Effects of insulin resistance and type 2 diabetes on lipoprotein subclass particle size and concentration determined by nuclear magnetic resonance. Diabetes. 2003;52(2):453-462.

16. Superko HR, Pendyala L, Williams PT, Momary KM, King SB, Garrett BC. High-density lipoprotein subclasses and their relationship to cardiovascular disease. J Clin Lipidol. 2012;6(6):496-523.

17. Hafiane A, Genest J. High density lipoproteins: Measurement techniques and potential biomarkers of cardiovascular risk. $B B A$ Clin. 2015;3:175-188.

18. Ståhlman M, et al. Dyslipidemia, but not hyperglycemia and insulin resistance, is associated with marked alterations in the HDL lipidome in type 2 diabetic subjects in the DIWA cohort: impact on small HDL particles. Biochim Biophys Acta. 2013;1831(11):1609-1617.

19. Hung ND, Sok DE, Kim MR. Prevention of 1-palmitoyl lysophosphatidylcholine-induced inflammation by polyunsaturated acyl lysophosphatidylcholine. Inflamm Res. 2012;61(5):473-483.

20. Sutter I, et al. Plasmalogens of high-density lipoproteins (HDL) are associated with coronary artery disease and anti-apoptotic activity of HDL. Atherosclerosis. 2015;241(2):539-546.

21. Khan AA, et al. Weight Loss and Exercise Alter the High-Density Lipoprotein Lipidome and Improve High-Density Lipoprotein Functionality in Metabolic Syndrome. Arterioscler Thromb Vasc Biol. 2018;38(2):438-447.

22. Denimal D, et al. Major changes in the sphingophospholipidome of HDL in non-diabetic patients with metabolic syndrome. Atherosclerosis. 2016;246:106-114.

23. Meikle PJ, et al. HDL Phospholipids, but Not Cholesterol Distinguish Acute Coronary Syndrome From Stable Coronary Artery Disease. J Am Heart Assoc. 2019;8(11):e011792.

24. Lee JH, Yang JS, Lee SH, Moon MH. Analysis of lipoprotein-specific lipids in patients with acute coronary syndrome by asymmetrical flow field-flow fractionation and nanoflow liquid chromatography-tandem mass spectrometry. J Chromatogr B Analyt Technol Biomed Life Sci. 2018;1099:56-63.

25. Kunz F, Pechlaner C, Erhart R, Fend F, Mühlberger V. HDL and plasma phospholipids in coronary artery disease. Arterioscler Thromb. 1994;14(7):1146-1150.

26. Getz GS, Krishack PA, Reardon CA. Serum amyloid A and atherosclerosis. Curr Opin Lipidol. 2016;27(5):531-535.

27. Zewinger S, et al. Serum amyloid A: high-density lipoproteins interaction and cardiovascular risk. Eur Heart J. 2015;36(43):3007-3016.

28. Dittrich J, et al. Plasma levels of apolipoproteins C-III, A-IV, and E are independently associated with stable atherosclerotic cardiovascular disease. Atherosclerosis. 2019;281:17-24.

29. Kronenberg F, et al. Low apolipoprotein A-IV plasma concentrations in men with coronary artery disease. J Am Coll Cardiol. 2000;36(3):751-757.

30. Ezeh B, et al. Plasma distribution of apoA-IV in patients with coronary artery disease and healthy controls. J Lipid Res. 2003;44(8):1523-1529.

31. Riwanto $\mathrm{M}$, et al. Altered activation of endothelial anti- and proapoptotic pathways by high-density lipoprotein from patients with coronary artery disease: role of high-density lipoprotein-proteome remodeling. Circulation. 2013;127(8):891-904.

32. Emmens JE, et al. Proteomic diversity of high-density lipoprotein explains its association with clinical outcome in patients with heart failure. Eur J Heart Fail. 2018;20(2):260-267.

33. Kopecky C, et al. Quantification of HDL proteins, cardiac events, and mortality in patients with type 2 diabetes on hemodialysis. Clin J Am Soc Nephrol. 2015;10(2):224-231.

34. Brahimaj A, et al. Serum Levels of Apolipoproteins and Incident Type 2 Diabetes: A Prospective Cohort Study. Diabetes Care. 2017;40(3):346-351. 
35. Rhainds D, Tardif JC. From HDL-cholesterol to HDL-function: cholesterol efflux capacity determinants. Curr Opin Lipidol. 2019;30(2):101-107.

36. Sorci-Thomas MG, Thomas MJ. Microdomains, Inflammation, and Atherosclerosis. Circ Res. 2016;118(4):679-691.

37. Nofer JR. Signal transduction by HDL: agonists, receptors, and signaling cascades. Handb Exp Pharmacol. 2015;224:229-256.

38. Jin Z, et al. Development and Validation of Apolipoprotein AI-Associated Lipoprotein Proteome Panel for the Prediction of Cholesterol Efflux Capacity and Coronary Artery Disease. Clin Chem. 2019;65(2):282-290.

39. Pamir N, et al. Genetic control of the mouse HDL proteome defines HDL traits, function, and heterogeneity. J Lipid Res. 2019;60(3):594-608.

40. Low-Kam C, et al. Variants at the APOE /C1/C2/C4 Locus Modulate Cholesterol Efflux Capacity Independently of High-Density Lipoprotein Cholesterol. J Am Heart Assoc. 2018;7(16):e009545.

41. Anastasius M, Luquain-Costaz C, Kockx M, Jessup W, Kritharides L. A critical appraisal of the measurement of serum 'cholesterol efflux capacity' and its use as surrogate marker of risk of cardiovascular disease. Biochim Biophys Acta Mol Cell Biol Lipids. 2018;1863(10):1257-1273.

42. Nicholls SJ, et al. Cholesterol Efflux Capacity and Pre-Beta-1 HDL Concentrations Are Increased in Dyslipidemic Patients Treated With Evacetrapib. J Am Coll Cardiol. 2015;66(20):2201-2210.

43. Han S, et al. Inhibition of cholesteryl ester transfer protein by anacetrapib does not impair the anti-inflammatory properties of high density lipoprotein. Biochim Biophys Acta. 2013;1831(4):825-833.

44. Othman A, et al. Plasma C20-Sphingolipids predict cardiovascular events independently from conventional cardiovascular risk factors in patients undergoing coronary angiography. Atherosclerosis. 2015;240(1):216-221.

45. Polito AJ, Akita T, Sweeley CC. Gas chromatography and mass spectrometry of sphingolipid bases. Characterization of sphinga-4,14-dienine from plasma sphingomyelin. Biochemistry. 1968;7(7):2609-2614.

46. Renkonen O, Hirvisalo EL. Structure of plasma sphingadienine. J Lipid Res. 1969;10(6):687-693.

47. Chew WS, et al. Large-scale lipidomics identifies associations between plasma sphingolipids and T2DM incidence. JCI Insight. 2019;5:126925.

48. Proia RL, Hla T. Emerging biology of sphingosine-1-phosphate: its role in pathogenesis and therapy. J Clin Invest. 2015;125(4):1379-1387.

49. Ruiz M, Okada H, Dahlbäck B. HDL-associated ApoM is anti-apoptotic by delivering sphingosine 1-phosphate to S1P1 \& S1P3 receptors on vascular endothelium. Lipids Health Dis. 2017;16(1):36.

50. Deeg MA, Bierman EL, Cheung MC. GPI-specific phospholipase D associates with an apoA-I- and apoA-IV-containing complex. J Lipid Res. 2001;42(3):442-451.

51. Raikwar NS, Cho WK, Bowen RF, Deeg MA. Glycosylphosphatidylinositol-specific phospholipase D influences triglyceride-rich lipoprotein metabolism. Am J Physiol Endocrinol Metab. 2006;290(3):E463-E470.

52. Raikwar NS, Bowen-Deeg RF, Du XS, Low MG, Deeg MA. Glycosylphosphatidylinositol-specific phospholipase D improves glucose tolerance. Metab Clin Exp. 2010;59(10):1413-1420.

53. Masuda S, et al. Impact of glycosylphosphatidylinositol-specific phospholipase D on hepatic diacylglycerol accumulation, steatosis, and insulin resistance in diet-induced obesity. Am J Physiol Endocrinol Metab. 2019;316(2):E239-E250.

54. von Toerne C, et al. MASP1, THBS1, GPLD1 and ApoA-IV are novel biomarkers associated with prediabetes: the KORA F4 study. Diabetologia. 2016;59(9):1882-1892.

55. Qin W, Liang YZ, Qin BY, Zhang JL, Xia N. The Clinical Significance of Glycoprotein Phospholipase D Levels in Distinguishing Early Stage Latent Autoimmune Diabetes in Adults and Type 2 Diabetes. PLoS ONE. 2016;11(6):e0156959.

56. Wang X, Driscoll DM, Morton RE. Molecular cloning and expression of lipid transfer inhibitor protein reveals its identity with apolipoprotein F. J Biol Chem. 1999;274(3):1814-1820.

57. Lagor WR, et al. Overexpression of apolipoprotein F reduces HDL cholesterol levels in vivo. Arterioscler Thromb Vasc Biol. 2009;29(1):40-46.

58. Lagor WR, et al. The effects of apolipoprotein F deficiency on high density lipoprotein cholesterol metabolism in mice. PLoS ONE. 2012;7(2):e31616.

59. Morton RE, Liu Y, Izem L. ApoF knockdown increases cholesteryl ester transfer to LDL and impairs cholesterol clearance in fat-fed hamsters. J Lipid Res. 2019;60(11):1868-1879.

60. Lagor WR, et al. Genetic manipulation of the ApoF/Stat2 locus supports an important role for type I interferon signaling in atherosclerosis. Atherosclerosis. 2014;233(1):234-241.

61. Morton RE, Nunes V, Izem L, Quintão E. Markedly elevated lipid transfer inhibitor protein in hypercholesterolemic subjects is mitigated by plasma triglyceride levels. Arterioscler Thromb Vasc Biol. 2001;21(10):1642-1649.

62. Morton RE, Gnizak HM, Greene DJ, Cho KH, Paromov VM. Lipid transfer inhibitor protein (apolipoprotein F) concentration in normolipidemic and hyperlipidemic subjects. J Lipid Res. 2008;49(1):127-135.

63. Ohnsorg PM, et al. Carboxyl terminus of apolipoprotein A-I (ApoA-I) is necessary for the transport of lipid-free ApoA-I but not prelipidated ApoA-I particles through aortic endothelial cells. J Biol Chem. 2011;286(10):7744-7754.

64. Wessel D, Flügge UI. A method for the quantitative recovery of protein in dilute solution in the presence of detergents and lipids. Anal Biochem. 1984;138(1):141-143.

65. Liebisch G, Lieser B, Rathenberg J, Drobnik W, Schmitz G. High-throughput quantification of phosphatidylcholine and sphingomyelin by electrospray ionization tandem mass spectrometry coupled with isotope correction algorithm. Biochim Biophys Acta. 2004;1686(1-2):108-117.

66. Liebisch G, Binder M, Schifferer R, Langmann T, Schulz B, Schmitz G. High throughput quantification of cholesterol and cholesteryl ester by electrospray ionization tandem mass spectrometry (ESI-MS/MS). Biochim Biophys Acta. 2006;1761(1):121-128.

67. Ritsch A, Scharnagl H, März W. HDL cholesterol efflux capacity and cardiovascular events. N Engl J Med. 2015;372(19):1870-1871.

68. Janjic D, Maechler P, Sekine N, Bartley C, Annen AS, Wolheim CB. Free radical modulation of insulin release in INS-1 cells exposed to alloxan. Biochem Pharmacol. 1999;57(6):639-648.

69. Elabd C, et al. Human multipotent adipose-derived stem cells differentiate into functional brown adipocytes. Stem Cells. 2009;27(11):2753-2760. 
70. Buuren S van, Groothuis-Oudshoorn K. mice: Multivariate Imputation by Chained Equations in R. Journal of Statistical Software. 2011;45(3):1-67.

71. Mertens BJA. Transformation, Normalization, and Batch Effect in the Analysis of Mass Spectrometry Data for Omics Studies. In: Mertens BJA, Datta S, eds. Statistical Analysis of Proteomics, Metabolomics, and Lipidomics Data Using Mass Spectrometry. Cham, Switzerland: Springer International; 2017:1-21. Frontiers in Probability and the Statistical Sciences.

72. Benjamini Y, Hochberg Y. Controlling the False Discovery Rate: A Practical and Powerful Approach to Multiple Testing Journal of the Royal Statistical Society: Series B (Methodological). 1995;57(1):289-300.

73. Bates D, Mächler M, Bolker B, Walker S. Fitting Linear Mixed-Effects Models Using lme4. Journal of Statistical Software. 2015;67(1):1-48.

74. Zou H, Hastie T. Regularization and variable selection via the elastic net. Journal of the Royal Statistical Society: Series B (Statistical Methodology). 2005;67(2):301-320.

75. Meinshausen N, Bühlmann P. Stability selection. Journal of the Royal Statistical Society: Series B (Statistical Methodology). 2010;72(4):417-473.

76. Shah RD, Samworth RJ. Variable selection with error control: another look at stability selection. Journal of the Royal Statistical Society: Series B (Statistical Methodology). 2013;75(1):55-80.

77. Wold S, Sjöström M, Eriksson L. PLS-regression: a basic tool of chemometrics. Chemometrics and Intelligent Laboratory Systems. 2001;58(2):109-130.

78. Chun H, Keleş S. Sparse partial least squares regression for simultaneous dimension reduction and variable selection. $J R$ Stat Soc Series B Stat Methodol. 2010;72(1):3-25.

79. Lauritzen SL. Graphical Models. Oxford, United Kingdom: Oxford University Press; 1996. Oxford Statistical Science Series.

80. Friedman J, Hastie T, Tibshirani R. Sparse inverse covariance estimation with the graphical lasso. Biostatistics. 2008;9(3):432-441.

81. Liu H, Lafferty J, Wasserman L. The Nonparanormal: Semiparametric Estimation of High Dimensional Undirected Graphs. Journal of Machine Learning Research. 2009;10:2295-2328.

82. Liu H, Roeder K, Wasserman L. Stability Approach to Regularization Selection (StARS) for High Dimensional Graphical Models. Paper presented at: Neural Informations Processing Systems Conference; December 6-11, 2010; Vancouver, British Columbia, Canada. http://papers.nips.cc/paper/3966-stability-approach-to-regularization-selection-stars-for-high-dimensional-graphical-models . Accessed December 11, 2019 